\title{
Quasiequilibrium sequences of synchronized and irrotational binary neutron stars in general relativity. I. Method and tests
}

\author{
Eric Gourgoulhon, Philippe Grandclément, Keisuke Taniguchi, Jean-Alain Marck and Silvano Bonazzola \\ Département d'Astrophysique Relativiste et de Cosmologie \\ UMR 8629 du C.N.R.S., Observatoire de Paris, \\ F-92195 Meudon Cedex, France
}

(15 December 2000)

\begin{abstract}
We present a numerical method to compute quasiequilibrium configurations of close binary neutron stars in the pre-coalescing stage. A hydrodynamical treatment is performed under the assumption that the flow is either rigidly rotating or irrotational. The latter state is technically more complicated to treat than the former one (synchronized binary), but is expected to represent fairly well the late evolutionary stages of a binary neutron star system. As regards the gravitational field, an approximation of general relativity is used, which amounts to solving five of the ten Einstein equations (conformally flat spatial metric). The obtained system of partial differential equations is solved by means of a multi-domain spectral method. Two spherical coordinate systems are introduced, one centered on each star; this results in a precise description of the stellar interiors. Thanks to the multi-domain approach, this high precision is extended to the strong field regions. The computational domain covers the whole space so that exact boundary conditions are set to infinity. Extensive tests of the numerical code are performed, including comparisons with recent analytical solutions. Finally a constant baryon number sequence (evolutionary sequence) is presented in details for a polytropic equation of state with $\gamma=2$.
\end{abstract}

PACS number(s): 04.25.Dm, 04.40.Dg, 97.60.Jd, 97.80.-d, 02.70.Hm

\section{INTRODUCTION}

Inspiraling neutron star binaries are expected to be among the strongest sources of gravitational radiation that could be detected by the interferometric detectors currently under construction (GEO600, LIGO, and VIRGO) or in operation (TAMA300). Such binary systems are therefore subject to numerous theoretical studies (see e.g. [1] for a review). Among them there are (i) Post-Newtonian (PN) analytical treatments (e.g. [2], [3], [雨) and (ii) fully relativistic hydrodynamical treatments, pioneered by the works of Oohara \& Nakamura (see e.g. [5]), Wilson et al. [6] ] ] and recently developed by Shibata [8 11], the Neutron Star Grand Challenge group [12,13] and Oohara \& Nakamura [14]. These last three groups integrate forward in time the evolution equations resulting from the $3+1$ formulation of general relativity 15.16]. In parallel of these dynamical calculations, some quasiequilibrium formulation of the problem has been developed [17 20] and successfully implemented 21 24. The basic assumption underlying the quasiequilibrium calculations is that the timescale of the orbit shrinking is larger than that of the orbital revolution in the pre-coalescing state. Consequently the evolution of the binary system can be approximated by a succession of exactly circular orbits, hence the name quasiequilibrium. The study of these quasiequilibrium configurations is justified in the view that the fully dynamical computations mentioned above are only in their infancy. In particular, they cannot follow more than a few orbits. Also they involve a rather coarse resolution of the stars, being performed in a single box with Cartesian coordinates. Another motivation for computing quasiequilibrium configurations is to provide valuable initial conditions for the dynamical evolutions 11, 12, 14.

The first quasiequilibrium configurations of binary neutron stars in general relativity have been obtained three years ago by Baumgarte et al. 25, 26], followed by Marronetti et al. [27]. However these computations considered synchronized binaries. This rotation state does not correspond to physical situations, since it has been shown that the gravitational-radiation driven evolution is too rapid for the viscous forces to synchronize the spin of each neutron star with the orbit [28,29] as they do for ordinary stellar binaries. Rather, the viscosity is negligible and the fluid velocity circulation (with respect to some inertial frame) is conserved in these systems. Provided that the initial spins are not in the millisecond regime, this means that close binary configurations are well approximated by zero vorticity (i.e. irrotational) states. Irrotational configurations are more complicated to obtain because the fluid velocity does not vanish in the co-orbiting frame (as it does for synchronized binaries). We have successfully developed a numerical method to tackle this problem and presented the first quasiequilibrium configurations of irrotational binary neutron stars elsewhere [21]. The numerical technique relies on a multi-domain spectral method [30] within spherical coordinates. Since then, two other groups have obtained relativistic irrotational configurations: (i) Marronetti, 
Mathews \& Wilson 22,31 by means of single-domain finite difference method within Cartesian coordinates and (ii) Uryu, Eriguchi and Shibata [23,24 by means of multi-domain finite difference method within spherical coordinates.

The present article is devoted to the detailed presentation of our method, along with numerous tests of the numerical code, while the previous letter [21] gave only a sketch of the equations and some results about an evolutionary sequence built on a polytropic equation of state. In particular, that letter focuses on the evolution of the central density along the sequence in order to investigate the stability of each star against gravitational collapse. That study was motivated by the 1995 finding of Wilson et al. [6, 7] that the neutron stars may individually collapse into a black hole prior to merger. This unexpected result has been called into question by a number of authors (see Ref. [32] for a summary of all the criticisms and some answers). Recently Flanagan 33 has found an error in the analytical formulation used by Wilson et al. [6,7]. New numerical computations by Mathews and Wilson [34], using a corrected code, show a significantly reduced compression effect.

The plan of the article is as follows. We start by presenting the equations governing binary stars in general relativity in Sect. II (hydrodynamics) and Sect. III (gravitational field). The numerical method developed to integrate these equations is presented in Sect. IV. Section $\mathrm{V}$ is then devoted to the tests passed by the numerical code. Astrophysical results are then presented in Sect. VI for an evolutionary sequence of irrotational binary stars constructed on polytropic equation of state of adiabatic index $\gamma=2$. Section VII contains the final discussion (comparison of our method with that used by other groups, conclusions about the tests) and future prospects.

Throughout the present article, we use units of $G=c=1$ where $G$ and $c$ denote the gravitational constant and speed of light.

\section{RELATIVISTIC EQUATIONS GOVERNING BINARIES IN CIRCULAR ORBITS}

Our treatment of binary neutron stars relies on the assumptions of (i) quasiequilibrium state (i.e. steady state in the co-orbiting frame), (ii) a specific velocity state for the fluid: either rigid or irrotational flow, (iii) the spatial 3-metric is almost conformally flat. In this section, we examine the assumptions (i) and (ii), without invoking assumption (iii), which will be introduced only in Sect. III.

\section{A. Quasiequilibrium assumption}

In the late inspiral phase, before any orbital instability or merging of the two stars, the evolution of binary neutron stars can be approximated by a succession of circular orbits. Indeed when the separation between the centers of the two neutron stars is about $50 \mathrm{~km}$ (in harmonic coordinates) the time variation of the orbital period, $\dot{P}_{\text {orb }}$, computed at the 2nd Post-Newtonian (PN) order by means of the formulas established by Blanchet et al. [35] is about $2 \%$. The evolution at this stage can thus be still considered as a sequence of equilibrium configurations. Moreover the orbits are expected to be circular (vanishing eccentricity), as a consequence of the gravitational radiation reaction [36]. In terms of the spacetime geometry, we translate these assumptions by demanding that there exists a Killing vector field 1 which is expressible as 17

$$
\mathbf{l}=\mathbf{k}+\Omega \mathbf{m}
$$

where $\Omega$ is a constant, to be identified with the orbital angular velocity with respect to a distant inertial observer, and $\mathbf{k}$ and $\mathbf{m}$ are two vector fields with the following properties: $\mathbf{k}$ is timelike at least far from the binary and is normalized so that far from the star it coincides with the 4-velocity of inertial observers with respect to which the total ADM 3-momentum of the system vanishes. On the other hand $\mathbf{m}$ is a spacelike vector field which has closed orbits and is zero on a two dimensional timelike surface, called the rotation axis. $\mathbf{m}$ is normalized so that $\nabla(\mathbf{m} \cdot \mathbf{m}) \cdot \nabla(\mathbf{m} \cdot \mathbf{m}) /(4 \mathbf{m} \cdot \mathbf{m})$ tends to 1 on the rotation axis [this latter condition ensures that the parameter $\varphi$ associated with $\mathbf{m}$ along its trajectories by $\mathbf{m}=\partial / \partial \varphi$ has the standard $2 \pi$ periodicity]. Let us call $\mathbf{l}$ the helicoidal Killing vector. We assume that $\mathbf{l}$ is a symmetry generator not only for the spacetime metric $\mathbf{g}$ but also for all the matter fields. In particular, 1 is tangent to the world tubes representing the surface of each star, hence its qualification of helicoidal (cf. Figure 1 of Ref. [17]).

The approximation suggested above amounts to neglecting outgoing gravitational radiation. For non-axisymmetric systems - as binaries are - imposing $\mathbf{l}$ as an exact Killing vector leads to a spacetime which is not asymptotically flat [37]. Thus, in solving for the gravitational field equations, a certain approximation has to be devised in order to avoid the divergence of some metric coefficients at infinity. For instance such an approximation could be the Wilson \& Mathews scheme [38] that amounts to solving only for the Hamiltonian and momentum constraint equations, as well as the trace of the spatial part of the "dynamical" Einstein equations (cf. Sect. III A). This approximation has 
been used in all the relativistic quasiequilibrium studies to date and is consistent with the existence of the helicoidal Killing vector field (11). Note also that since the gravitational radiation reaction shows up only at the $2.5-\mathrm{PN}$ order, the helicoidal symmetry is exact up to the 2-PN order.

Following the standard $3+1$ formalism [15], we introduce a foliation of spacetime by a family of spacelike hypersurfaces $\Sigma_{t}$ such that at spatial infinity, the vector $\mathbf{k}$ introduced in Eq. (1) is normal to $\Sigma_{t}$ and the ADM 3-momentum in $\Sigma_{t}$ vanishes (i.e. the time $t$ is the proper time of an asymptotic inertial observer at rest with respect to the binary system). Asymptotically, $\mathbf{k}=\partial / \partial t$ and $\mathbf{m}=\partial / \partial \varphi$, where $\varphi$ is the azimuthal coordinate associated with the above asymptotic inertial observer, so that Eq. (11) can be re-written as

$$
\mathbf{l}=\frac{\partial}{\partial t}+\Omega \frac{\partial}{\partial \varphi} .
$$

One can then introduce the shift vector $\mathbf{B}$ of co-orbiting coordinates by means of the orthogonal decomposition of $\mathbf{l}$ with respect to the $\Sigma_{t}$ foliation:

$$
\mathbf{l}=N \mathbf{n}-\mathbf{B}
$$

where $\mathbf{n}$ is the unit future directed vector normal to $\Sigma_{t}, N$ is called the lapse function and $\mathbf{n} \cdot \mathbf{B}=0$.

\section{B. Fluid motion}

We consider a perfect fluid, which constitutes an excellent approximation for neutron star matter. The matter stress-energy tensor is then

$$
\mathbf{T}=(e+p) \mathbf{u} \otimes \mathbf{u}+p \mathbf{g}
$$

$e$ being the fluid proper energy density, $p$ the fluid pressure, $\mathbf{u}$ the fluid 4-velocity, and $\mathbf{g}$ the spacetime metric. A zero-temperature equation of state (EOS) is a very good approximation for neutron star matter. For such an EOS, the first law of thermodynamics gives rise to the following identity (Gibbs-Duhem relation):

$$
\frac{\nabla p}{e+p}=\frac{1}{h} \nabla h,
$$

where $h$ is the fluid specific enthalpy:

$$
h:=\frac{e+p}{m_{\mathrm{B}} n}
$$

$n$ being the fluid baryon number density and $m_{\mathrm{B}}$ the mean baryon mass: $m_{\mathrm{B}}=1.66 \times 10^{-27} \mathrm{~kg}$. Note that for our zero-temperature EOS, $m_{\mathrm{B}} h$ is equal to the fluid chemical potential.

By means of the identity (5), it is straightforward to show that the classical momentum-energy conservation equation $\nabla \cdot \mathbf{T}=0$ is equivalent to the set of two equations [39,40]:

$$
\begin{gathered}
\mathbf{u} \cdot(\nabla \wedge \mathbf{w})=0, \\
\nabla \cdot(n \mathbf{u})=0 .
\end{gathered}
$$

In Eq. (7), w is the co-momentum 1-form

$$
\mathbf{w}:=h \mathbf{u}
$$

and $\nabla \wedge \mathbf{w}$ denotes the exterior derivative of $\mathbf{w}$, i.e. the vorticity 2 -form [39]. In terms of components, one has

$$
(\nabla \wedge \mathbf{w})_{\alpha \beta}=\nabla_{\alpha} w_{\beta}-\nabla_{\beta} w_{\alpha}=\partial_{\alpha} w_{\beta}-\partial_{\beta} w_{\alpha}
$$

The vorticity 2-form enters Cartan's identity which states that the Lie derivative of the 1-form w along the vector field $\mathbf{l}$ is

$$
£_{\mathbf{l}} \mathbf{w}=\mathbf{l} \cdot(\nabla \wedge \mathbf{w})+\nabla(\mathbf{l} \cdot \mathbf{w}) .
$$


Because of the assumed helicoidal symmetry, $£_{\mathbf{l}} \mathbf{w}=0$, so that Cartan's identity reduces to

$$
\mathbf{l} \cdot(\nabla \wedge \mathbf{w})+\nabla(\mathbf{l} \cdot \mathbf{w})=0 .
$$

This equation reveals to be very useful in the following; this justifies the introduction of the vorticity 2-form.

In particular, performing the scalar product of Eq. (12) by the fluid 4-velocity $\mathbf{u}$ leads to

$$
\mathbf{l} \cdot(\nabla \wedge \mathbf{w}) \cdot \mathbf{u}+\mathbf{u} \cdot \nabla(\mathbf{l} \cdot \mathbf{w})=0 .
$$

The first term in the left-hand side vanishes by virtue of the equation of motion (7), so that we obtain

$$
\mathbf{u} \cdot \nabla(\mathbf{l} \cdot \mathbf{w})=0
$$

which means that the quantity $\mathbf{l} \cdot \mathbf{w}=h \mathbf{l} \cdot \mathbf{u}$ is constant along each streamline. This is the relativistic generalization of the classical Bernoulli theorem. At this stage, it must be noticed that, in order for the constant to be uniform over the streamlines, i.e., to be a constant over spacetime, so that one gets a first integral for the fluid motion, some additional property of the flow must be required. In the following two sections, we explore two such additional properties: rigidity and irrotationality.

\section{Rigid rotation}

A rigid motion corresponds to synchronized stars (also called corotating stars). It is defined in relativity by the vanishing of the expansion tensor $\theta_{\alpha \beta}:=\left(g_{\alpha}{ }^{\mu}+u_{\alpha} u^{\mu}\right)\left(g_{\beta}{ }^{\nu}+u_{\beta} u^{\nu}\right) \nabla_{(\nu} u_{\mu)}$ of the 4 -velocity $\mathbf{u}$. In the presence of a Killing vector $\mathbf{l}$, this can be realized by requiring the colinearity of $\mathbf{u}$ and $\mathbf{l}$ :

$$
\mathbf{u}=\lambda \mathbf{l},
$$

where $\lambda$ is a scalar field related to the norm of $\mathbf{l}$ by the normalization of the 4 -velocity $\lambda=(-\mathbf{l} \cdot \mathbf{l})^{-1 / 2}$. Inserting relation (15) into the equation of fluid motion (1) shows that the first term in Eq. (12) vanishes identically, so that one gets the well known first integral of motion [4]

$$
\mathbf{l} \cdot \mathbf{w}=\text { const. }
$$

The second part of the equations of fluid motion, Eq. (8) (baryon number conservation), is trivially satisfied by the form (15) because $\mathbf{l}$ is a Killing vector.

\section{Irrotational flow}

As recalled in Sect. I, realistic binary neutron stars are not expected to be in synchronized rotation, but rather to have an irrotational motion. A relativistic irrotational flow is defined by the vanishing of the vorticity 2-form [39] :

$$
\nabla \wedge \mathbf{w}=0 .
$$

This is equivalent to the existence of a scalar field $\Psi$ such that

$$
\mathbf{w}=\nabla \Psi \text {. }
$$

This is the relativistic definition of a potential flow [42]. Note that the advantage of writing the equation for the fluid motion in the form (7)-(8) rather than in the traditional form $\nabla \cdot \mathbf{T}=0$ is that one can see immediately that a flow of the form (18) is a solution of (7).

The second part of the equation of motion, Eq. (8), is satisfied by the potential flow (18) provided that $\Psi$ obeys to the equation

$$
\frac{n}{h} \nabla \cdot \nabla \Psi+\nabla \Psi \cdot \nabla\left(\frac{n}{h}\right)=0
$$

Inserting the irrotationality condition (17) into Eq. (12) results in an equation showing the constancy of the scalar product $\mathbf{l} \cdot \mathbf{w}$ :

$$
\mathbf{l} \cdot \mathbf{w}=\text { const. }
$$

We therefore obtain the same first integral as in the rigid case (Eq. (16) above). However note that the way to get it is different: no use of the equation of motion (7) has been made to obtain (20), contrary to the derivation of Eq. (16). The first integral (16) for rigid motion has been known for a long time, at least since Boyer's work [41. To our knowledge, the version (20) for an irrotational flow in presence of a Killing vector is due to Carter [40]. 


\section{E. $3+1$ decomposition}

The first integral $(16),(20)$, common to both the rigid and irrotational motion, is expressed in terms of the contraction of a spacetime vector (1) with a spacetime 1 -form (w). Going back to the $3+1$ formalism mentioned in Sect. II A, let us re-express it in terms of quantities relative to the hypersurfaces $\Sigma_{t}$. Following Ref. [17], we introduce the co-orbiting observer, whose 4 -velocity $\mathbf{v}$ is the normalized symmetry generator:

$$
\mathbf{v}=\left(N^{2}-\mathbf{B} \cdot \mathbf{B}\right)^{-1 / 2} \mathbf{l}
$$

where the normalization factor has been deduced from Eq. (3). Note that in the rigid motion case, the co-orbiting observer and the fluid comoving observer coincide: $\mathbf{u}=\mathbf{v}$ [cf. Eq. (15)]. The $3+1$ split of the 4 -velocity $\mathbf{v}$ with respect to the Eulerian observer is

$$
\mathbf{v}=\Gamma_{0}\left(\mathbf{n}+\mathbf{U}_{0}\right)
$$

where

$$
\Gamma_{0}=-\mathbf{n} \cdot \mathbf{v}=\left(1-\mathbf{U}_{0} \cdot \mathbf{U}_{0}\right)^{-1 / 2}
$$

is the Lorentz factor between the two observers and $\mathbf{U}_{0}$ is the orbital 3-velocity with respect to the Eulerian observer $\left(\mathbf{n} \cdot \mathbf{U}_{0}=0\right)$. According to Eqs. 21) and (3), $\mathbf{U}_{0}$ is linked to the shift vector of co-orbiting coordinates by

$$
\mathbf{U}_{0}=-\frac{\mathbf{B}}{N}
$$

Thanks to the second part of Eq. (23), Eq. (21) can be re-written as

$$
\mathbf{v}=\frac{\Gamma_{0}}{N} \mathbf{l}
$$

The fluid motion can be described by the following orthogonal decompositions of $\mathbf{u}$ :

$$
\mathbf{u}=\Gamma(\mathbf{v}+\mathbf{V})=\Gamma_{\mathrm{n}}(\mathbf{n}+\mathbf{U})
$$

where $\Gamma=-\mathbf{v} \cdot \mathbf{u}$ (resp. $\left.\Gamma_{\mathrm{n}}=-\mathbf{n} \cdot \mathbf{u}\right)$ is the Lorentz factor between the fluid and the co-orbiting (resp. Eulerian)

observer, and $\mathbf{V}$ (resp. $\mathbf{U}$ ) is the fluid 3-velocity with respect to the co-orbiting (resp. Eulerian) observer. In particular, $\mathbf{v} \cdot \mathbf{V}=0, \mathbf{n} \cdot \mathbf{U}=0$ and

$$
\mathbf{U}=\frac{1}{\Gamma_{\mathrm{n}}} \mathbf{h} \cdot \mathbf{u}
$$

where

$$
\mathbf{h}:=\mathbf{g}+\mathbf{n} \otimes \mathbf{n}
$$

is the orthogonal projector onto the spatial hypersurfaces $\Sigma_{t} ; \mathbf{h}$ can also be viewed as the metric induced by $\mathbf{g}$ onto the hypersurfaces $\Sigma_{t}$. Performing the scalar product of Eq. 22) with the second part of Eq. (26) leads to an expression of the Lorentz factor $\Gamma$ in terms of quantities relative to the Eulerian observer only:

$$
\Gamma=\Gamma_{\mathrm{n}} \Gamma_{0}\left(1-\mathbf{U} \cdot \mathbf{U}_{0}\right)
$$

Similarly, performing the projection of the second part of Eq. (26) onto the hyperplane orthogonal to $\mathbf{v}$ results in the expression of the fluid 3-velocity $\mathbf{V}$ with respect to the co-orbiting observer in terms of the 3-velocities $\mathbf{U}$ and $\mathbf{U}_{0}$, both defined with respect to the Eulerian observer:

$$
\mathbf{V}=\frac{\Gamma_{0}}{1-\mathbf{U} \cdot \mathbf{U}_{0}}\left[\mathbf{U}_{0} \cdot\left(\mathbf{U}-\mathbf{U}_{0}\right) \mathbf{n}+\mathbf{U}-\mathbf{U}_{0}+\left(\mathbf{U} \cdot \mathbf{U}_{0}\right) \mathbf{U}_{0}-\left(\mathbf{U}_{0} \cdot \mathbf{U}_{0}\right) \mathbf{U}\right] .
$$

Note that in the case where $\mathbf{U}$ and $\mathbf{U}_{0}$ are aligned $\left(\mathbf{U}=U \mathbf{e}\right.$ and $\mathbf{U}_{0}=U_{0} \mathbf{e}$, e being some unit vector in $\left.\Sigma_{t}\right)$ relation (30) reduces to the classical velocity-addition law of special relativity: $\mathbf{V}=\left(U-U_{0}\right) /\left(1-U U_{0}\right) \mathbf{e}^{\prime}$, where $\mathbf{e}^{\prime}=\Gamma_{0}\left(\mathbf{e}+U_{0} \mathbf{n}\right)$ is the unit vector deduced from e by a boost of velocity $U_{0}$. In particular for $\mathbf{U}=\mathbf{U}_{0}$, which corresponds to synchronized binaries, $\mathbf{V}$ vanishes identically. 
For irrotational binaries, $\mathbf{U}$ is related to the potential $\Psi$ by combining Eqs. (9), (18) and (27) :

$$
\mathbf{U}=\frac{1}{\Gamma_{\mathrm{n}} h} \mathbf{D} \Psi
$$

where $\mathbf{D}$ is the covariant derivative associated with the metric $\mathbf{h}$ of spatial hypersurfaces $\Sigma_{t}$. Combined with the relation $\Gamma_{\mathrm{n}}=(1-\mathbf{U} \cdot \mathbf{U})^{-1 / 2}$, this relation results in

$$
\Gamma_{\mathrm{n}}=\left(1+\frac{1}{h^{2}} \mathbf{D} \Psi \cdot \mathbf{D} \Psi\right)^{1 / 2}
$$

We are now in position to write the $3+1$ form of the first integral $(16),(20)$, common to both the rigid and irrotational motion. Substituting relation (9) for $\mathbf{w}$ and relation (25) for 1 into Eq. (16) results in

$$
h N \frac{\Gamma}{\Gamma_{0}}=\text { const } .
$$

We shall use actually the logarithm of this relation:

$$
H+\nu-\ln \Gamma_{0}+\ln \Gamma=\text { const }
$$

with the following definitions:

$$
H:=\ln h
$$

and

$$
\nu:=\ln N
$$

These two quantities have immediate meaning at the Newtonian limit: $H$ is the (non-relativistic) specific enthalpy and $\nu$ is the Newtonian gravitational potential. The first integral of motion written as (34) coincides with Eq. (66) of Ref. [17]. The link with the alternative expressions derived by Teukolsky [19] and by Shibata 20] for the irrotational case is performed in Appendix A. Note that $\ln \Gamma=0$ for synchronized binaries, so that Eq. (34) simplifies somewhat. Note also that substituting Eq. (29) for $\Gamma$ in Eq. (34) leads to an alternative expression of the first integral of motion which contains only quantities relative to the Eulerian observer:

$$
H+\nu+\ln \Gamma_{\mathrm{n}}+\ln \left(1-\mathbf{U} \cdot \mathbf{U}_{0}\right)=\text { const } .
$$

However in the following, we shall use only the form (34).

Let us now turn to the $3+1$ form the differential equation (19) for the velocity potential $\Psi$ of irrotational flows. Taking into account the helicoidal symmetry, Eq. (19) becomes

$$
n \mathbf{D} \cdot \mathbf{D} \Psi+\mathbf{D} n \cdot \mathbf{D} \Psi=h \Gamma_{\mathrm{n}} \mathbf{U}_{0} \cdot \mathbf{D} n+n\left(\mathbf{D} \Psi \cdot \mathbf{D} \ln \frac{h}{N}+\mathbf{U}_{0} \cdot \mathbf{D} \Gamma_{\mathrm{n}}\right)+n h K \Gamma_{\mathrm{n}}
$$

where $K$ is the trace of the extrinsic curvature tensor of the $\Sigma_{t}$ hypersurfaces. This equation has been obtained by Teukolsky [19 and independently by Shibata [20]. We refer to these authors for the details of the derivation of Eq. (38) from Eq. (19).

\section{F. Newtonian limit}

At the Newtonian limit, the Eulerian observer is an inertial observer. Eqs. (2) and (3) show that $\mathbf{B}=-\Omega \frac{\partial}{\partial \varphi}$, so that Eq. (24) for the velocity of the co-orbiting observer with respect to the inertial observer becomes

$$
\mathbf{U}_{0}=\Omega \times \mathbf{r}
$$

where $\mathbf{r}$ denotes the position vector with respect to the center of mass of the system. The logarithm of the corresponding Lorentz factor tends to (minus) the centrifugal potential [cf. Eq. (23)] 


$$
\ln \Gamma_{0}=\frac{1}{2}(\Omega \times \mathbf{r})^{2} .
$$

The Newtonian limit of the first integral of motion (34) for synchronized binaries $(\ln \Gamma=0)$ gives the classical expression

$$
H+\nu-\frac{1}{2}(\Omega \times \mathbf{r})^{2}=\text { const },
$$

where, as recalled above, $H$ is the fluid specific enthalpy and $\nu$ the Newtonian gravitational potential.

In the irrotational case, the Newtonian limit results in the following fluid velocity with respect to the inertial frame [set $h=1$ and $\Gamma_{\mathrm{n}}=1$ in Eq. (31)]

$$
\mathbf{U}=\vec{\nabla} \Psi
$$

where $\vec{\nabla}$ denotes the standard 3-dimensional gradient operator, i.e. the Newtonian limit of the operator $\mathbf{D}$ introduced above. The first integral of motion (37) reduces then to

$$
H+\nu+\frac{1}{2}(\vec{\nabla} \Psi)^{2}-(\Omega \times \mathbf{r}) \cdot \vec{\nabla} \Psi=\text { const. }
$$

We recognize the classical expression (compare e.g. with Eq. (12) of Ref. [19] or Eq. (11) of Ref. [43]). The Newtonian limit of the continuity equation (38) reads

$$
n \Delta \Psi+\vec{\nabla} n \cdot \vec{\nabla} \Psi=(\Omega \times \mathbf{r}) \cdot \vec{\nabla} n .
$$

Here again, we recognize the classical expression (compare e.g. with Eq. (13) of Ref. [19]).

\section{GRAVITATIONAL FIELD EQUATIONS}

\section{A. A simplifying assumption: the conformally flat 3-metric}

As a first step in the treatment of binary configurations in general relativity, a simplifying assumption can be introduced, in order to reduce the computational task, namely to take the 3-metric induced in the hypersurfaces $\Sigma_{t}$ to be conformally flat:

$$
\mathbf{h}=A^{2} \mathbf{f}
$$

where $A$ is some scalar field and $\mathbf{f}$ is a flat 3-metric. This assumption has been first introduced by Wilson \& Mathews [38] and has been employed in all the studies of quasiequilibrium relativistic binaries to date 21 26]. It has been also used in binary black hole initial data computations (see e.g. 44 47]). It is physically less justified than the assumption of quasiequilibrium discussed above. However, some possible justifications of (45) are

1. it is exact for spherically symmetric configurations;

2. it is very accurate for axisymmetric rotating neutron stars 48;

3. the 1-PN metric fits it;

4. the 2.5-PN metric [49] deviates from it by only $2 \%$ for two $1.4 M_{\odot}$ neutron stars as close as $30 \mathrm{~km}$ (in harmonic coordinates) [50].

\section{B. Partial differential equations for the metric}

To benefit from the helicoidal symmetry, we use co-orbiting coordinates $\left(t, x^{1}, x^{2}, x^{3}\right)$, i.e. coordinates adapted to the Killing vector $\mathbf{l}: \partial / \partial t=\mathbf{l}$. Assuming the conformally flat form (45) for $\mathbf{h}$, the full spacetime metric takes then the form円

\footnotetext{
${ }^{1}$ Latin indices $i, j, \ldots$ run from 1 to 3 .
} 


$$
d s^{2}=-\left(N^{2}-B_{i} B^{i}\right) d t^{2}-2 B_{i} d t d x^{i}+A^{2} f_{i j} d x^{i} d x^{j}
$$

We have thus five metric functions to determine: the lapse $N$, the conformal factor $A$ and the three components $B^{i}$ of the shift vector $\mathbf{B}$ [see Eq. (3)]. Let us define auxiliary metric quantities: we have already introduced the logarithm of $N, \nu$, via Eq. (36); we introduce now the shift vector of non-rotating coordinates:

$$
\mathbf{N}=\mathbf{B}+\Omega \frac{\partial}{\partial \varphi}
$$

and the quantity

$$
\beta:=\ln (A N)
$$

At the Newtonian limit $\mathbf{N}=0$ and $\beta=0$.

In the following, we choose the slicing of spacetime by the hypersurfaces $\Sigma_{t}$ to be maximal. This results in $K=0$.

The Killing equation $\nabla_{\alpha} l_{\beta}+\nabla_{\beta} l_{\alpha}=0$, gives rise to a relation between the $\Sigma_{t}$ extrinsic curvature tensor $\mathbf{K}$ and the shift vector $\mathbf{N}$ (via Eq. (3) and the relation $\nabla \mathbf{n}=-\mathbf{K}-\mathbf{n} \otimes \mathbf{D} \ln N$ )

$$
K^{i j}=-\frac{1}{2 N}\left(D^{i} B^{j}+D^{j} B^{i}\right)=-\frac{1}{2 A^{2} N}\left\{\bar{\nabla}^{i} N^{j}+\bar{\nabla}^{j} N^{i}-\frac{2}{3} f^{i j} \bar{\nabla}_{k} N^{k}\right\}
$$

where $\bar{\nabla}$ stands for the covariant derivative associated with the flat 3-metric $\mathbf{f}$. Here and in the following, the index $i$ of $\bar{\nabla}^{i}$ is supposed to be raised with the metric f. Note that since $\partial / \partial \phi$ is a Killing vector of the flat metric f, the second part of this equation stands also with $N^{i}$ replaced by $B^{i}$.

The trace of the spatial part of the Einstein equation, combined with the Hamiltonian constraint equation, result in the following two equations

$$
\begin{gathered}
\underline{\Delta} \nu=4 \pi A^{2}(E+S)+A^{2} K_{i j} K^{i j}-\bar{\nabla}_{i} \nu \bar{\nabla}^{i} \beta, \\
\underline{\Delta}=4 \pi A^{2} S+\frac{3}{4} A^{2} K_{i j} K^{i j}-\frac{1}{2}\left(\bar{\nabla}_{i} \nu \bar{\nabla}^{i} \nu+\bar{\nabla}_{i} \beta \bar{\nabla}^{i} \beta\right),
\end{gathered}
$$

whereas the momentum constraint equation yields, by means of Eq. (49),

$$
\underline{\Delta} N^{i}+\frac{1}{3} \bar{\nabla}^{i}\left(\bar{\nabla}_{j} N^{j}\right)=-16 \pi N A^{2}(E+p) U^{i}+2 N A^{2} K^{i j} \bar{\nabla}_{j}(3 \beta-4 \nu) .
$$

In these equations, $\underline{\Delta}:=\bar{\nabla}^{i} \bar{\nabla}_{i}$ is the Laplacian operator associated with the flat metric $\mathbf{f}$, and $E$ and $S$ are respectively the matter energy density and trace of the stress tensor, both as measured by the Eulerian observer:

$$
\begin{gathered}
E:=\mathbf{n} \cdot \mathbf{T} \cdot \mathbf{n}=\Gamma_{\mathrm{n}}^{2}(e+p)-p, \\
S:=\mathbf{h} \cdot \mathbf{T}=3 p+(E+p) \mathbf{U} \cdot \mathbf{U} .
\end{gathered}
$$

The equations to be solved to get the metric coefficients are the elliptic equations (50)-(52). Note that they represent only five of the ten Einstein equations. The remaining five Einstein equations are not used in this procedure. Moreover, some of these remaining equations are certainly violated, reflecting the fact that the conformally flat 3-metric (45) is an approximation to the exact metric generated by a binary system.

At the Newtonian limit, Eqs. (51) and (52) reduce to $0=0$. There remains only Eq. (50), which gives the usual Poisson equation for the gravitational potential $\nu$.

\section{Equations for the fluid with a conformally flat 3-metric}

In this section we explicitly write some equations for fluid quantities when the 3-metric takes the form (45). First the Lorentz factor (23) between the co-orbiting and Eulerian observers writes

$$
\Gamma_{0}=\left(1-A^{2} f_{i j} U_{0}^{i} U_{0}^{j}\right)^{-1 / 2} .
$$


For irrotational motion, the expression (32) for the Lorentz factor $\Gamma_{\mathrm{n}}$ between the fluid and Eulerian observers becomes

$$
\Gamma_{\mathrm{n}}=\left(1+\frac{1}{A^{2} h^{2}} f^{i j} \bar{\nabla}_{i} \Psi \bar{\nabla}_{j} \Psi\right)^{1 / 2}
$$

The corresponding fluid 3-velocity 31 is

$$
U^{i}=\frac{1}{A^{2} \Gamma_{\mathrm{n}} h} \bar{\nabla}^{i} \Psi
$$

The Lorentz factor $\Gamma$ between the fluid and co-orbiting observer, which enters in the first integral of motion (34), is deduced from the above quantities via Eq. (29) :

$$
\Gamma=\Gamma_{\mathrm{n}} \Gamma_{0}\left(1-A^{2} f_{i j} U^{i} U_{0}^{j}\right) .
$$

Let us now consider the continuity equation (38). For a zero-temperature EOS, $H$ can be considered as a function of the baryon density $n$ solely, so that one can introduce the thermodynamical coefficient

$$
\zeta:=\frac{d \ln H}{d \ln n}
$$

The gradient of $n$ which appears in Eq. (38) can be then replaced by a gradient of $H$ so that, using the metric 45), one obtains

$$
\zeta H \triangleq \Psi+\bar{\nabla}^{i} H \bar{\nabla}_{i} \Psi=A^{2} h \Gamma_{\mathrm{n}} U_{0}^{i} \bar{\nabla}_{i} H+\zeta H\left(\bar{\nabla}^{i} \Psi \bar{\nabla}_{i}(H-\beta)+A^{2} h U_{0}^{i} \bar{\nabla}_{i} \Gamma_{\mathrm{n}}\right) .
$$

The potential $\Psi$ is in fact dominated by a pure translational part. Therefore, we write, in each star,

$$
\Psi=: \Psi_{0}+f_{i j} W_{0}^{i} x^{j}
$$

where $W_{0}^{i}$ is the constant (translational) velocity field defined as the central? value of

$$
W^{i}:=A^{2} h \Gamma_{\mathrm{n}} U_{0}^{i}
$$

Then $\bar{\nabla}^{i} \Psi=\bar{\nabla}^{i} \Psi_{0}+W_{0}^{i}$ and $\underline{\Psi}=\underline{\Delta} \Psi_{0}$, so that Eq. (60) becomes

$$
\zeta H \triangleq \Psi_{0}+\left[(1-\zeta H) \bar{\nabla}^{i} H+\zeta H \bar{\nabla}^{i} \beta\right] \bar{\nabla}_{i} \Psi_{0}=\left(W^{i}-W_{0}^{i}\right) \bar{\nabla}_{i} H+\zeta H\left(W_{0}^{i} \bar{\nabla}_{i}(H-\beta)+\frac{W^{i}}{\Gamma_{\mathrm{n}}} \bar{\nabla}_{i} \Gamma_{\mathrm{n}}\right) .
$$

The advantage to solve Eq. (63) instead of Eq. (60) is that the right-hand side of the former is much smaller than the right-hand side of the latter, due to the factor $\bar{W}^{i}-W_{0}^{i}$, instead of $W^{i}$, in front of $\bar{\nabla}_{i} H$.

\section{Global quantities}

The total mass-energy content in a $\Sigma_{t}$ hypersurface is given by the Arnowitt-Deser-Misner (ADM) mass $M$, which is expressed by means of the surface integral at spatial infinity

$$
M=\frac{1}{16 \pi} \oint_{\infty} f^{i k} f^{j l}\left(\bar{\nabla}_{j} h_{k l}-\bar{\nabla}_{k} h_{j l}\right) d S_{i}
$$

(see e.g. Eq. (20.9) of Ref. [51]). In the case of the conformally flat 3 -metric $h_{i j}=A^{2} f_{i j}$, this integral can be written

$$
M=-\frac{1}{2 \pi} \oint_{\infty} \bar{\nabla}^{i} A^{1 / 2} d S_{i}
$$

\footnotetext{
${ }^{2}$ The centers of the stars are defined in Sect. IV A.
} 
By means of the Gauss-Ostragradsky formula, this expression can be converted into the volume integral of $\underline{\Delta} A^{1 / 2}$. This last quantity can be expressed by subtracting Eq. (50) from Eq. (51) (recall that $A=\exp (\beta-\nu)$ ), so that Eq. (65) becomes an integral containing the matter energy density and the extrinsic curvature of $\Sigma_{t}$ :

$$
M=\int_{\Sigma_{t}} A^{5 / 2}\left(E+\frac{1}{16 \pi} K_{i j} K^{i j}\right) d^{3} x
$$

Following Bowen \& York [52] we define the total angular momentum in a $\Sigma_{t}$ hypersurface as the surface integral at spatial infinity

$$
J_{i}=\frac{1}{16 \pi} \epsilon_{i j k} \oint_{\infty}\left(x^{j} K^{k l}-x^{k} K^{j l}\right) d S_{l}=\frac{1}{16 \pi} \epsilon_{i j k} \oint_{\infty}\left(x^{j} A^{5} K^{k l}-x^{k} A^{5} K^{j l}\right) d S_{l},
$$

where $\epsilon_{i j k}$ is the 3 -dimensional alternating tensor, $x^{i}$ are Cartesian coordinates, and the second equality follows from the fact that $A=1$ at spatial infinity. As for $M$, this integral can be converted to a volume integral on $\Sigma_{t}$. Using the momentum constraint equation $D_{l} K^{k l}=8 \pi(E+p) U^{k}$ and the fact that $D_{l} K^{k l}=A^{-5} \partial\left(A^{5} K^{k l}\right) / \partial x^{l}$ for the conformally flat 3 -metric (45), one obtains the expression

$$
J_{i}=\epsilon_{i j k} \int_{\Sigma_{t}} A^{5}(E+p) x^{j} U^{k} d^{3} x .
$$

The baryon mass of each star is given by the integral on $\Sigma_{t}$ of the baryon number density as measured by the Eulerian observer: $-n \mathbf{u} \cdot \mathbf{n}=\Gamma_{\mathrm{n}} n$. In the case of the conformally flat 3-metric (45), this integral becomes

$$
M_{\mathrm{B}}^{\langle a\rangle}=m_{\mathrm{B}} \int_{\text {star } a} A^{3} \Gamma_{\mathrm{n}} n d^{3} x, a=1,2 .
$$

\section{NUMERICAL METHOD}

The equations to be solved to get a relativistic binary system in quasiequilibrium are the elliptic equations (50)-(52) for the gravitational field, supplemented by the elliptic equation (63) for the velocity potential $\Psi_{0}$ in the irrotational case. A cold matter equation of state, of the form

$$
n=n(H) \quad e=e(H) \quad p=p(H),
$$

must be supplied to close the system of equations. The thermodynamical quantity $H$ has been privileged in the EOS setting (70) because it is that quantity which is involved in the first integral of motion (34). Altogether, these equations constitute a system of coupled non-linear partial differential equations. We solve this system by means of an iterative procedure.

\footnotetext{
${ }^{3}$ Note that contrary to the ADM mass, the total angular momentum hence defined is not asymptotically gauge invariant: it is defined merely as the $1 / r^{3}$ part of $K_{i j}$ within our coordinates; see York 53] for a discussion.
} 


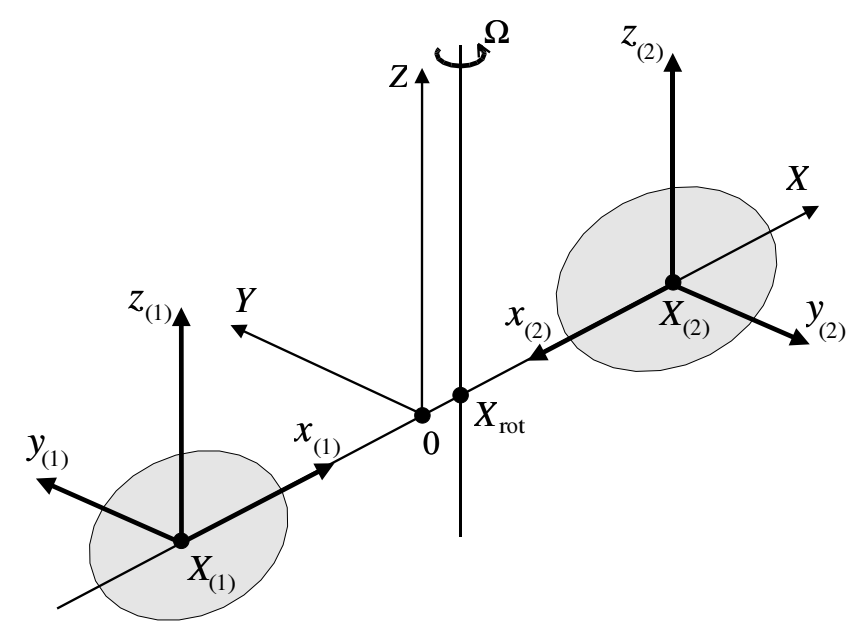

FIG. 1. Coordinate systems used in the calculation.

\section{A. Coordinate systems and computational domains}

We use co-orbiting coordinates $(t, X, Y, Z)$ of Cartesian type (i.e. $\left.f_{i j}=\delta_{i j}\right)$, so that the line element (46) can be written

$$
d s^{2}=-N^{2} d t^{2}+A^{2}\left[\left(d X-B^{X} d t\right)^{2}+\left(d Y-B^{Y} d t\right)^{2}+\left(d Z-B^{Z} d t\right)^{2}\right] .
$$

In these coordinates, the two stars have fixed locations and figures. Let us define the center of star no. $a(a=1,2)$ as the location of the maximum enthalpy $H$ (or equivalently maximum density $e$ ) in star $a$. Note that this center does not coincide with the center of mass of star $a$. We choose the coordinates $(X, Y, Z)$ such that (i) the orbital plane is defined by $Z=0$, (ii) the two stellar centers are located along the $X$ axis and (iii) the rotation axis (cf. Sect. IIA) is located at $X=0, Y=0$. Let us then denote by $X_{\langle 1\rangle}$ and $X_{\langle 2\rangle}$ the $X$ coordinates of the two stellar centers ${ }^{\mathbb{t}}$.

In order to describe properly the stellar interiors, we introduce two systems of Cartesian coordinates $\left(x_{\langle a\rangle}, y_{\langle a\rangle}, z_{\langle a\rangle}\right)$ centered on the two stars by (see Fig. 1)

$$
\left\{\begin{array} { l } 
{ x _ { \langle 1 \rangle } = X - X _ { \langle 1 \rangle } } \\
{ y _ { \langle 1 \rangle } = Y } \\
{ z _ { \langle 1 \rangle } = Z }
\end{array} \quad \text { and } \quad \left\{\begin{array}{l}
x_{\langle 2\rangle}=-\left(X-X_{\langle 2\rangle}\right) \\
y_{\langle 2\rangle}=-Y \\
z_{\langle 2\rangle}=Z
\end{array}\right.\right.
$$

Note that the system $\left(x_{\langle 1\rangle}, y_{\langle 1\rangle}, z_{\langle 1\rangle}\right)$ is aligned with $(X, Y, Z)$, whereas $\left(x_{\langle 2\rangle}, y_{\langle 2\rangle}, z_{\langle 2\rangle}\right)$ is anti-aligned (rotation of angle $\pi$ in the $(X, Y)$ plane) with $(X, Y, Z)$. This choice ensures that the companion of star no. $a$ is located at $x_{\langle a\rangle}>0$ for both stars. In particular, for equal mass stars, the descriptions of each star in terms of $\left(x_{\langle a\rangle}, y_{\langle a\rangle}, z_{\langle a\rangle}\right)$ are identical. Furthermore we introduce spherical coordinates $\left(r_{\langle a\rangle}, \theta_{\langle a\rangle}, \varphi_{\langle a\rangle}\right)(a=1,2)$ associated with each of the Cartesian coordinate systems $\left(x_{\langle a\rangle}, y_{\langle a\rangle}, z_{\langle a\rangle}\right)$ by means of the usual formulæ.

Since some of the equations to be solved are elliptic equations with non-compactly supported sources, the computational domain must extend up to spatial infinity, i.e. must cover the full hypersurface $\Sigma_{t}$, in order to put correct boundary conditions (flat spacetime). Any truncated computational domain ("box") would result in approximate boundary conditions, which inevitably would induce some error in the numerical solution. The technique to cover the full $\Sigma_{t}$ is to divide it in various domains, the outermost of it being compactified in order to deal with finite computational domain only [30]. Following the introduction of the two coordinate systems $\left(r_{\langle a\rangle}, \theta_{\langle a\rangle}, \varphi_{\langle a\rangle}\right)$ (one centered on each star), we will actually use two sets of such domains: one centered on star no. 1, the other on star no. 2 . The number $N_{\langle a\rangle}$ of domains in each set is arbitrary, being simply equal or larger than 3 . The list of the $N_{\langle 1\rangle}+N_{\langle 2\rangle}$ computational domains is

\footnotetext{
${ }^{4}$ In all this article, indices or superscripts in \langle\rangle will label the two stars.
} 


$$
\begin{array}{lllll}
\mathcal{D}_{0}^{\langle 1\rangle} & \ldots & \mathcal{D}_{M_{\langle 1\rangle}-1}^{\langle 1\rangle} & \ldots & \mathcal{D}_{N_{\langle 1\rangle}-1}^{\langle 1\rangle} \\
\mathcal{D}_{0}^{\langle 2\rangle} & \ldots & \mathcal{D}_{M_{\langle 2\rangle}-1}^{\langle 2\rangle} & \ldots & \mathcal{D}_{N_{\langle 2\rangle}-1}^{\langle 2\rangle}
\end{array}
$$

where

- Domain $\mathcal{D}_{0}^{\langle a\rangle}(a=1,2)$ has the topology of a ball and contains the center of star $a$; it is designed thereafter as the nucleus.

- $M_{\langle a\rangle}(a=1,2)$ is the number of domains which cover the interior of star $a$. It obeys $M_{\langle a\rangle} \geq 1$ and $M_{\langle a\rangle} \leq N_{\langle a\rangle}-2$. The outer boundary of domain $\mathcal{D}_{M_{\langle a\rangle}-1}^{\langle a\rangle}$ coincides exactly with the surface of star $a$. The topology of domains $\mathcal{D}_{1}^{\langle a\rangle}, \ldots, \mathcal{D}_{M_{\langle a\rangle}-1}^{\langle a\rangle}$ is that of a spherical shell; these domains are designed thereafter as the shells.

- Domains $\mathcal{D}_{M_{\langle a\rangle}}^{\langle a\rangle}, \ldots, \mathcal{D}_{N_{\langle a\rangle}-2}^{\langle a\rangle}$ cover the non-compactified part of the space outside star $a$; they are also called shells. The inner boundary of domain $\mathcal{D}_{M_{\langle a\rangle}}^{\langle a\rangle}$ coincides exactly with the surface of star $a$.

- Domain $\mathcal{D}_{N_{\langle a\rangle}-1}^{\langle a\rangle}$ is the most external one; it extends up to $r=+\infty$. We call this domain the compactified external domain (CED) since thanks to some compactification it will be mapped to a finite computational domain.

Of course the two sets of domains overlap since $\mathcal{D}_{0}^{\langle 1\rangle} \cup \ldots \cup \mathcal{D}_{N_{\langle 1\rangle}-1}^{\langle 1\rangle}=\mathcal{D}_{0}^{\langle 2\rangle} \cup \ldots \cup \mathcal{D}_{N_{\langle 2\rangle}-1}^{\langle 2\rangle}=\Sigma_{t}$. The various domains are represented in Fig. 2 1 for $N_{\langle 1\rangle}=N_{\langle 2\rangle}=3$.

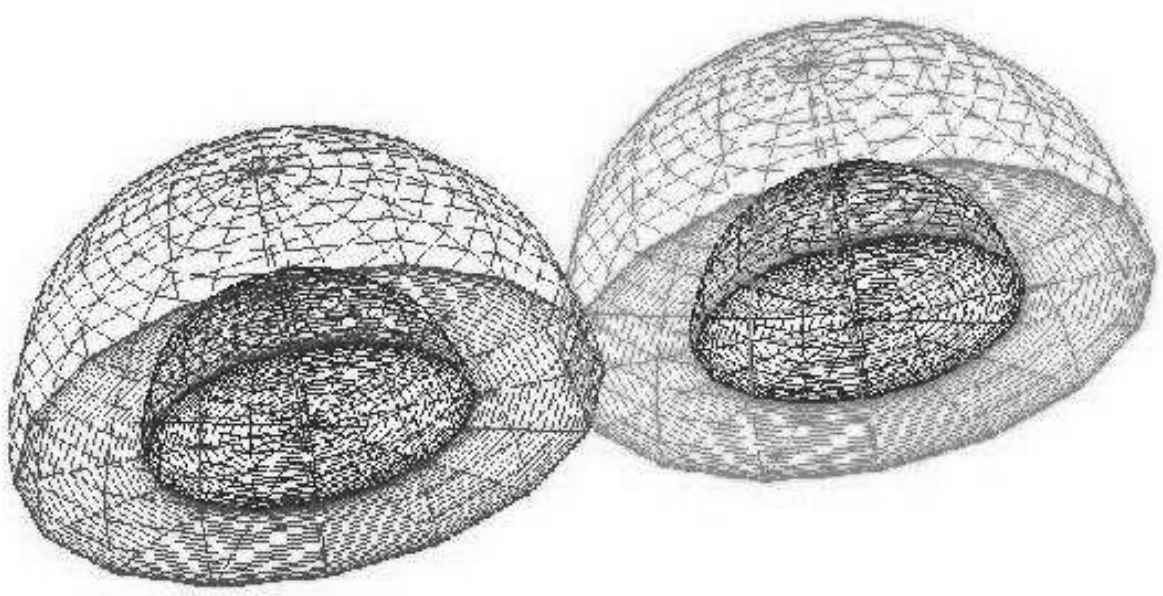

FIG. 2. Domains used in the numerical computations, when $N_{\langle 1\rangle}=N_{\langle 2\rangle}=3$. The boundaries of domains $\mathcal{D}_{0}^{\langle 1\rangle}, \mathcal{D}_{1}^{\langle 1\rangle}, \mathcal{D}_{0}^{\langle 2\rangle}$ and $\mathcal{D}_{1}^{\langle 2\rangle}$ are represented. The outer boundaries of domains $\mathcal{D}_{2}^{\langle 1\rangle}$ and $\mathcal{D}_{2}^{\langle 2\rangle}$ are located at infinity and are therefore not plotted.

Following the technique introduced previously [30], we define in each domain the computational coordinates $\left(\xi, \theta^{\prime}, \varphi^{\prime}\right)$ according to

$$
\theta^{\prime}=\theta, \quad \varphi^{\prime}=\varphi
$$

and

- in the nucleus:

$$
r=\alpha_{0}\left[\xi+\left(3 \xi^{4}-2 \xi^{6}\right) F_{0}(\theta, \varphi)+\frac{1}{2}\left(5 \xi^{3}-3 \xi^{5}\right) G_{0}(\theta, \varphi)\right], \quad \xi \in[0,1]
$$

\footnotetext{
${ }^{5}$ For the sake of clarity we omit here the star indices $\langle a\rangle$ on the spherical coordinates $\left(r_{\langle a\rangle}, \theta_{\langle a\rangle}, \varphi_{\langle a\rangle}\right)$ centered on star $a$.
} 
- in the shells $\left(1 \leq l \leq N_{\langle a\rangle}-2\right)$ :

$$
r=\alpha_{l}\left[\xi+\frac{1}{4}\left(\xi^{3}-3 \xi+2\right) F_{l}(\theta, \varphi)+\frac{1}{4}\left(-\xi^{3}+3 \xi+2\right) G_{l}(\theta, \varphi)\right]+\beta_{l}, \quad \xi \in[-1,1]
$$

- in the CED:

$$
r=\frac{2 R_{\mathrm{CED}}}{1-\xi}, \quad \xi \in[-1,1]
$$

In the above relations, $\alpha_{l}$ and $\beta_{l}$ are some constants, the functions $F_{l}(\theta, \varphi)$ and $G_{l}(\theta, \varphi)$ define the boundary of each domain: the outer boundary of the nucleus corresponds to $\xi=1$ and is given by the equation

$$
r=\alpha_{0}\left[1+F_{0}(\theta, \varphi)+G_{0}(\theta, \varphi)\right]
$$

where $F_{0}(\theta, \varphi)$ contains only odd Fourier harmonics in $\varphi$ and $G_{0}(\theta, \varphi)$ only even harmonics, the inner boundary of the shell no. $l\left(1 \leq l \leq N_{\langle a\rangle}-2\right)$ corresponds to $\xi=-1$ and is given by the equation

$$
r=\alpha_{l}\left[-1+F_{l}(\theta, \varphi)\right]+\beta_{l},
$$

whereas its outer boundary corresponds to $\xi=1$ and is given by the equation

$$
r=\alpha_{l}\left[1+G_{l}(\theta, \varphi)\right]+\beta_{l} .
$$

Finally $R_{\mathrm{CED}}$ is the radius of the inner boundary of the CED, which is assumed to be spherical.

\section{B. Multi-domain spectral method}

In each domain, we expand the various physical fields in a series of basis functions of $\xi, \theta^{\prime}$ and $\varphi^{\prime}$. We use Chebyshev polynomials in $\xi$, trigonometrical polynomials or associated Legendre functions in $\theta^{\prime}$, and Fourier series in $\varphi^{\prime}$. The interested reader is referred to Sect. III.A of Ref. 30] for more details about these spectral expansions. Let us denote by $N_{r}^{\langle a\rangle}(l)$ the number of coefficient in the $\xi$ expansion used in domain $\mathcal{D}_{l}^{\langle a\rangle}$, by $N_{\theta}^{\langle a\rangle}(l)$ the number of coefficients in the $\theta^{\prime}$ expansion and by $N_{\varphi}^{\langle a\rangle}(l)$ the number of coefficients in the $\varphi^{\prime}$ expansion. We employ a collocation spectral method, which means that in each domain, a function can be described either by the coefficients of its spectral expansion or by its value at some particular grid points, called the collocation points [54. The grids plotted in Fig. 2 2 show actually these collocation points.

The spectral method amounts to reducing linear partial differential equations into a system of algebraic equations for the coefficients of the spectral expansions. We refer to [55,56] for the details of this multi-domain spectral method and here simply recall some basic features:

- As explained above, spherical-type coordinates $\left(\xi, \theta^{\prime}, \varphi^{\prime}\right)$ centered on each star are used: this ensures a much better description of the stars than by means of Cartesian coordinates.

- These spherical-type coordinates are surface-fitted coordinates: i.e. the surface of each star lies at a constant value of the coordinate $\xi$ thanks to the mapping $\left(\xi, \theta^{\prime}, \varphi^{\prime}\right) \mapsto(r, \theta, \varphi)$ defined by Eqs. (74)-(75). This ensures that the spectral method applied in each domain is free from any Gibbs phenomenon (spurious oscillations generated by discontinuities).

- The outermost domain extends up to spatial infinity, thanks to the mapping $(76)$. This enables us to put exact boundary conditions on the elliptic equations (50)-(52) for the metric coefficients: spatial infinity is the only location where the metric is known in advance (Minkowski metric).

- Thanks to the use of a spectral method in each domain, the numerical error is evanescent for analytical fields (e.g. density fields for a $\gamma=2$ equation of state), i.e. it decreases exponentially with the number of coefficients (or equivalently collocation grid points) used in the spectral expansions [55,56]. 


\section{Splitting of the metric quantities}

Having introduced two sets of computational domains (grids), we linearly split the metric potentials $\nu, \beta$ and $N^{i}$ into

$$
\begin{gathered}
\nu=\nu_{\langle 1\rangle}+\nu_{\langle 2\rangle}=\nu_{\langle 1\rangle}+\nu_{\langle 2 \rightarrow 1\rangle}=\nu_{\langle 1 \rightarrow 2\rangle}+\nu_{\langle 2\rangle}, \\
\beta=\beta_{\langle 1\rangle}+\beta_{\langle 2\rangle}=\beta_{\langle 1\rangle}+\beta_{\langle 2 \rightarrow 1\rangle}=\beta_{\langle 1 \rightarrow 2\rangle}+\beta_{\langle 2\rangle}, \\
N^{i}=N_{\langle 1\rangle}^{i}+N_{\langle 2\rangle}^{i}=N_{\langle 1\rangle}^{i}+N_{\langle 2 \rightarrow 1\rangle}^{i}=N_{\langle 1 \rightarrow 2\rangle}^{i}+N_{\langle 2\rangle}^{i},
\end{gathered}
$$

where the quantities labeled by " $\langle a\rangle$ " or " $\langle b \rightarrow a\rangle$ " $(a=1,2, b=3-a)$ are defined at the collocation points of the domains $\mathcal{D}_{l}^{\langle a\rangle}$ centered on star $a$, and the quantities labeled by " $\langle a\rangle$ " and " $\langle a \rightarrow b\rangle$ " represents the same physical field but described at different collocation points (those of domain sets $\mathcal{D}_{l}^{\langle a\rangle}$ and $\mathcal{D}_{l}^{\langle b\rangle}$ respectively), i.e. $\nu_{\langle 1 \rightarrow 2\rangle}=\nu_{\langle 1\rangle}$, $\nu_{\langle 2 \rightarrow 1\rangle}=\nu_{\langle 2\rangle}$, etc...

The basic idea underlying the splittings (80)-(82) is that for each metric potential, there are two primary quantities, those labeled by " $\langle 1\rangle$ " and " $\langle 2\rangle$ ", which are "mostly generated" by respectively star 1 and star 2 and which we called the auto-potentials [the precise definitions are given by Eqs. (83)-(85) below]. The auto-potentials are obtained by solving the gravitational field equations, on domains $\mathcal{D}_{l}^{\langle 1\rangle}$ for the " $\langle 1\rangle$ " potentials, and on $\mathcal{D}_{l}^{\langle 2\rangle}$ for the " $\langle 2\rangle$ " ones. The quantities labeled by " $\langle 1 \rightarrow 2\rangle$ " [resp. " $\langle 2 \rightarrow 1\rangle$ "] are then merely representations of the " $\langle 1\rangle$ " [resp. " $\langle 2\rangle "]$ auto-potentials at the collocation points associated with the companion star. For this reason, we shall call them the comp-potentials.

Following the splittings (80)-(82), the gravitational field equations (50)-(52) are themselves split in two parts. $\nu_{\langle 1\rangle}$ and $\nu_{\langle 2\rangle}$ are thus defined as the solutions of the two equations

$$
\underline{\Delta} \nu_{\langle a\rangle}=4 \pi A^{2}\left(E_{\langle a\rangle}+S_{\langle a\rangle}\right)+Q_{\langle a\rangle}+Q_{\langle b \rightarrow a\rangle}-\bar{\nabla}_{i} \nu_{\langle a\rangle}\left[\bar{\nabla}^{i} \beta_{\langle a\rangle}+\left(\bar{\nabla}^{i} \beta\right)_{\langle b \rightarrow a\rangle}\right], \quad a=1,2 \quad(b=3-a),
$$

whereas $\beta_{\langle 1\rangle}$ and $\beta_{\langle 2\rangle}$ are defined as the solutions of the two equations

$$
\begin{aligned}
\underline{\Delta}_{\langle a\rangle}= & 4 \pi A^{2} S_{\langle a\rangle}+\frac{3}{4}\left(Q_{\langle a\rangle}+Q_{\langle b \rightarrow a\rangle}\right)-\frac{1}{2} \bar{\nabla}_{i} \nu_{\langle a\rangle}\left[\bar{\nabla}^{i} \nu_{\langle a\rangle}+\left(\bar{\nabla}^{i} \nu\right)_{\langle b \rightarrow a\rangle}\right] \\
& -\frac{1}{2} \bar{\nabla}_{i} \beta_{\langle a\rangle}\left[\bar{\nabla}^{i} \beta_{\langle a\rangle}+\left(\bar{\nabla}^{i} \beta\right)_{\langle b \rightarrow a\rangle}\right], \quad a=1,2 \quad(b=3-a),
\end{aligned}
$$

and $N_{\langle 1\rangle}^{i}$ and $N_{\langle 2\rangle}^{i}$ are defined as the solutions of the two equations

$$
\begin{aligned}
\underline{\Delta} N_{\langle a\rangle}^{i}+\frac{1}{3} \bar{\nabla}^{i}\left(\bar{\nabla}_{j} N_{\langle a\rangle}^{j}\right)= & -16 \pi N A^{2}\left(E_{\langle a\rangle}+p_{\langle a\rangle}\right) U_{\langle a\rangle}^{i}+N \tilde{K}_{\langle a\rangle}^{i j}\left(6\left[\bar{\nabla}_{j} \beta_{\langle a\rangle}+\left(\bar{\nabla}_{j} \beta\right)_{\langle b \rightarrow a\rangle}\right]\right. \\
& \left.-8\left[\bar{\nabla}_{j} \nu_{\langle a\rangle}+\left(\bar{\nabla}_{j} \nu\right)_{\langle b \rightarrow a\rangle}\right]\right), \quad a=1,2 \quad(b=3-a) .
\end{aligned}
$$

In these equations, $E_{\langle a\rangle}, S_{\langle a\rangle}, p_{\langle a\rangle}, U_{\langle a\rangle}^{i}$ are the quantities relative to the fluid of star $a$ only and defined respectively by Eqs. (53), (54), (44) and (27). $\tilde{K}_{\langle a\rangle}^{i j}$ is defined from $N_{\langle a\rangle}^{i}$ according to

$$
\tilde{K}_{\langle a\rangle}^{i j}:=-\frac{1}{2 N}\left\{\bar{\nabla}^{i} N_{\langle a\rangle}^{j}+\bar{\nabla}^{j} N_{\langle a\rangle}^{i}-\frac{2}{3} f^{i j} \bar{\nabla}_{k} N_{\langle a\rangle}^{k}\right\}, \quad a=1,2,
$$

so that the total extrinsic curvature is given by $K^{i j}=\left(\tilde{K}_{\langle 1\rangle}^{i j}+\tilde{K}_{\langle 2\rangle}^{i j}\right) / A^{2}$. Finally $Q_{\langle a\rangle}$ and $Q_{\langle b \rightarrow a\rangle}$ are defined by

$$
\begin{gathered}
Q_{\langle a\rangle}:=A^{2} f_{i k} f_{j l} \tilde{K}_{\langle a\rangle}^{k l} \tilde{K}_{\langle a\rangle}^{i j}, \quad a=1,2, \\
Q_{\langle b \rightarrow a\rangle}:=A^{2} f_{i k} f_{j l} \tilde{K}_{\langle a\rangle}^{k l} \tilde{K}_{\langle b \rightarrow a\rangle}^{i j}, \quad a=1,2 \quad(b=3-a),
\end{gathered}
$$

where $\tilde{K}_{\langle b \rightarrow a\rangle}^{i j}$ is the same physical field than $\tilde{K}_{\langle b\rangle}^{i j}$ but numerically described at the collocation points of the domains $\mathcal{D}_{l}^{\langle a\rangle}, \tilde{K}_{\langle b\rangle}^{i j}$ being given at the collocation points of the domains $\mathcal{D}_{l}^{\langle b\rangle}$. 
It is straightforward to check that adding the two equations (83) results in Eq. (50), adding the two equations (84) results in Eq. (51) and adding the two equations (55) results in Eq. (52). Therefore, having obtained solutions $\nu_{\langle a\rangle}$, $\beta_{\langle a\rangle}$ and $N_{\langle a\rangle}^{i}$ of the equations (83)-(85), we can form the solution of the gravitational field equations (50), (51) and (52) via Eqs. (80)-(82).

The advantage of solving the system of $2 \times 5=10$ PDEs (83)-(85)), instead of solving the system of 5 PDEs (50)-(52), is that the source terms (right-hand-side) of the former are mostly concentrated on one of the two stars and therefore well described by one of the two domain sets introduced in Sect. IV A. This is not true for the source terms involving the comp-potentials " $\langle b \rightarrow a\rangle$ ". However these terms enter only via quadratic combinations in which each of them is multiplied by the gradient of an auto-potential term, which is small where the comp-potential is large, so that the product of the two is smaller than the other sources terms, such as the scalar product of gradients of auto-potentials. The same considerations hold for $Q_{\langle b \rightarrow a\rangle}$ which appears to be much smaller than $Q_{\langle a\rangle}$. According to these remarks, Eq. (83) for $\nu_{\langle 1\rangle}$ is naturally solved on domains $\mathcal{D}_{l}^{\langle 1\rangle}$, Eq. (83) for $\nu_{\langle 2\rangle}$ is solved on domains $\mathcal{D}_{l}^{\langle 2\rangle}$, and more generally, each equation for an auto-potential is solved onto the domains set centered on the corresponding star.

Once the auto-potentials are known (at a given step of the iterative procedure described in the next section), there remains to compute the corresponding comp-potentials. This means that given e.g. $\nu_{\langle 1\rangle}$ at the collocation points of domains $\mathcal{D}_{l}^{\langle 1\rangle}$, one has to compute its values $\nu_{\langle 1 \rightarrow 2\rangle}$ at the collocation points of domains $\mathcal{D}_{l}^{\langle 2\rangle}$. One may think first to use some interpolation technique since the two sets of domains overlap. But this will necessarily introduce some "numerical noise". We will proceed differently, taking advantage of the use of a spectral method. Indeed, the values of the field $\nu_{\langle 1\rangle}$ at the collocation points of domains $\mathcal{D}_{l}^{\langle 1\rangle}$ is not the only numerical representation of $\nu_{\langle 1\rangle}$ we have at our disposal. We can use the alternative representation by the set of coefficients of its spectral expansion in each domain $\mathcal{D}_{l}^{\langle 1\rangle}\left(0 \leq l \leq N_{\langle 1\rangle}-1\right)$ (cf. Sect. IVB). By means of this spectral expansion, we can compute the value of $\nu_{\langle 1\rangle}$ at any point in the domain $\mathcal{D}_{l}^{\langle 1\rangle}$, not necessarily a collocation point. Hence, given a collocation point $\left(\xi_{i}, \theta_{j}^{\prime}, \varphi_{k}^{\prime}\right)$ of domain $\mathcal{D}_{l_{0}}^{\langle 2\rangle}$, we first compute the corresponding physical spherical coordinates $\left(r_{\langle 2\rangle}, \theta_{\langle 2\rangle}, \varphi_{\langle 2\rangle}\right)$ via Eqs. (74)-( (76), then the corresponding Cartesian coordinates $\left(x_{\langle 2\rangle}, y_{\langle 2\rangle}, z_{\langle 2\rangle}\right)$; these latter are translated into Cartesian coordinates $\left(x_{\langle 1\rangle}, y_{\langle 1\rangle}, z_{\langle 1\rangle}\right)$ via Eq. (72). We finally obtain the corresponding spherical coordinates $\left(r_{\langle 1\rangle}, \theta_{\langle 1\rangle}, \varphi_{\langle 1\rangle}\right)$ centered on star 1 . We then determine in which domain $\mathcal{D}_{l}^{\langle 1\rangle}$ this point is localized and to which value of the coordinate $\xi$ it corresponds by inverting the relations $(74)-(76)$. Then we may use the spectral expansion of $\nu_{\langle 1\rangle}$ to get the searched value:

$$
\nu_{\langle 1 \rightarrow 2\rangle}\left(l_{0}, \xi_{i}, \theta_{j}^{\prime}, \varphi_{k}^{\prime}\right)=\sum_{k=0}^{N_{\varphi}^{\langle 1\rangle}(l)-1}\left[\sum_{j=0}^{N_{\theta}^{\langle 1\rangle}(l)-1}\left(\sum_{i=0}^{N_{r}^{\langle 1\rangle}(l)-1} \hat{\nu}_{l k j i} X_{k j i}(\xi)\right) \Theta_{k j}\left(\theta_{\langle 1\rangle}\right)\right] \Phi_{k}\left(\varphi_{\langle 1\rangle}\right)
$$

where the $\hat{\nu}_{l k j i}$ are the coefficients of $\nu_{\langle 1\rangle}$ in domain $\mathcal{D}_{l}^{\langle 1\rangle}, X_{k j i}$ denotes the basis functions in $\xi$ (typically Chebyshev polynomials), $\Theta_{k j}$ the basis functions in $\theta$ (typically $\cos n \theta$ or $\left.\sin n \theta\right)$ and $\Phi_{k}$ the basis functions in $\varphi$ (Fourier series). These functions depend on the type of domain (nucleus, shell or CED) and are described in details in Sect. III.A of Ref. [30].

\section{Iterative procedure}

Within our procedure, a quasiequilibrium binary neutron star configuration is obtained by specifying:

1. the equation of state 70 ) for each star;

2. the rotation state: either rigidly rotating (synchronized binaries, Sect. IIO) or irrotational flow (Sect. IID);

3. the coordinate distance $d:=\left|X_{\langle 2\rangle}-X_{\langle 1\rangle}\right|$ between the two stellar centers;

4. the central enthalpies $H_{\langle 1\rangle}^{\mathrm{c}}$ and $H_{\langle 2\rangle}^{\mathrm{c}}$ in each star, or equivalently, via (70), the central density in each star (with our definition of the stellar center, this coincides with the maximum density).

As we discuss below, item 4 can be replaced by the specification of the baryon mass of each star. 


\section{Initial conditions}

The above parameters being set, we start by computing initial conditions for the iterative procedure. These initial conditions are constituted by two numerical solutions for spherically symmetric static isolated neutron stars, of respective central enthalpy $H_{\langle 1\rangle}^{\mathrm{c}}$ and $H_{\langle 2\rangle}^{\mathrm{c}} \cdot M_{\langle 1\rangle}$ and $M_{\langle 2\rangle}$ being the gravitational masses of these spherical symmetric models, we set the $X$ coordinates of the two stellar centers according to the Newtonian-like formulas:

$$
X_{\langle 1\rangle}=-\frac{M_{\langle 2\rangle}}{M_{\langle 1\rangle}+M_{\langle 2\rangle}} d \quad \text { and } \quad X_{\langle 2\rangle}=\frac{M_{\langle 1\rangle}}{M_{\langle 1\rangle}+M_{\langle 2\rangle}} d .
$$

These coordinates will remain fixed during the iteration. Only the location of the rotation axis $X_{\text {rot }}$, initially set to 0 , will change (see Fig. 11). Accordingly the formulæ (90) have no physical meaning whatsoever. They can be viewed as the setting of the origin of the coordinate system $(X, Y, Z)$. The location of this origin is a priori arbitrary, only the distance $d$ between the two stellar centers having a physical meaning; the setting (90) simply insures that this origin is not too far from the rotation axis.

The angular velocity $\Omega$ is initialized according to a formula for second order Post-Newtonian spherical stars [35, 57]:

$$
\begin{aligned}
\Omega_{\text {ini }}^{2}=\frac{M_{\text {ini }}}{d^{3}}\{ & 1-\frac{M_{\text {ini }}}{d}\left[\frac{11}{4}+\frac{2 R^{2}}{d^{2}} \gamma-\frac{12}{25} \frac{R^{4}}{d^{4}} \gamma^{2}\right] \\
& \left.+\left(\frac{M_{\text {ini }}}{d}\right)^{2}\left[\frac{69}{8}+\frac{11}{4} \frac{R^{2}}{d^{2}} \gamma+\frac{17}{25} \frac{R^{4}}{d^{4}} \gamma^{2}\right]\right\},
\end{aligned}
$$

where $M_{\text {ini }}:=M_{\langle 1\rangle}+M_{\langle 2\rangle}, R$ is the coordinate radius of one of the two stars (which is spherical initially) and $\gamma=\gamma_{\text {irrot }}:=0$ for irrotational binaries, whereas $\gamma=\gamma_{\text {corot }}:=5 I_{\langle a\rangle} /\left(2 M_{\langle a\rangle} R_{\langle a\rangle}^{2}\right)$ for corotating binaries, $I_{\langle a\rangle}$ being the moment of inertia of star $a$. For this last quantity, we use as an ansatz the exact value for a Newtonian $n=1$ polytrope, which results in $\gamma_{\text {corot }}=5 / 3\left(1-6 / \pi^{2}\right)$, independent of $a$.

The metric auto-potentials are initialized as follows: $\nu_{\langle a\rangle}$ and $\beta_{\langle a\rangle}$ are set to the values of $\nu$ and $\beta$ for the static spherical models. The shift $N_{\langle a\rangle}^{i}$ is initialized to the first-order Post-Newtonian value for spherical incompressible binaries (this value can be obtained by taking the limit for a spherical star of the equations presented in Ref. [四)

$$
N_{\langle a\rangle}^{i}=\frac{7}{8} W_{\langle a\rangle}^{i}-\frac{1}{8}\left(\bar{\nabla}^{i} \chi_{\langle a\rangle}+\bar{\nabla}^{i} W_{\langle a\rangle}^{j} x_{j}\right), \quad a=1,2,
$$

with

$$
W_{\langle a\rangle}^{X}=0 ; \quad W_{\langle a\rangle}^{Y}= \begin{cases}\epsilon_{\langle a\rangle} \frac{6 M_{\langle a\rangle} \Omega_{\text {ini }} d}{\left(1+M_{\langle a\rangle} / M_{\langle b\rangle}\right) R_{\langle a\rangle}}\left(1-\frac{r_{\langle a\rangle}^{2}}{3 R_{\langle a\rangle}^{2}}\right) & \text { for } \quad r_{\langle a\rangle} \leq R_{\langle a\rangle} \quad ; \quad W_{\langle a\rangle}^{Z}=0, \\ \epsilon \frac{4 M_{\langle a\rangle} \Omega_{\text {ini }} d}{\epsilon_{\langle a\rangle}}\left(1+M_{\langle a\rangle} / M_{\langle b\rangle}\right) r_{\langle a\rangle} & \text { for } \quad r_{\langle a\rangle}>R_{\langle a\rangle}\end{cases}
$$

$\left(\epsilon_{\langle 1\rangle}:=-1, \epsilon_{\langle 2\rangle}:=1, b=3-a\right)$ and

$$
\chi_{\langle a\rangle}= \begin{cases}\frac{2 M_{\langle a\rangle} \Omega_{\mathrm{ini}} d}{\left(1+M_{\langle a\rangle} / M_{\langle b\rangle}\right) R_{\langle a\rangle}} y_{\langle a\rangle}\left(1-\frac{3 r_{\langle a\rangle}^{2}}{5 R_{\langle a\rangle}^{2}}\right) & \text { for } \quad r_{\langle a\rangle} \leq R_{\langle a\rangle} \\ \frac{4 M_{\langle a\rangle} \Omega_{\mathrm{ini}} d R_{\langle a\rangle}^{2}}{5\left(1+M_{\langle a\rangle} / M_{\langle b\rangle}\right)} \frac{y_{\langle a\rangle}}{r_{\langle a\rangle}^{3}} & \text { for } \quad r_{\langle a\rangle}>R_{\langle a\rangle}\end{cases}
$$

We refer to Sect. IVA for the definition of the coordinates $X, Y, Z, r_{\langle a\rangle}$ and $y_{\langle a\rangle}$ involved in these formulæ. It appeared that the initial shift vector given above results in a too large angular velocity in the first steps. Therefore, we artificially lower it by multiplying it by 0.6 . From this initial value of the shift, we get initial values of $\tilde{K}_{\langle a\rangle}^{i j}$ via Eq. (86), and initial values of $\mathbf{B}$ and $\mathbf{U}_{0}$ via Eqs. (47) and (24).

Regarding the fluid quantities, the 3-velocity $\mathbf{U}$ is initialized to $\mathbf{U}_{0}$ in the synchronized case, whereas in the irrotational case, $\Psi_{0}$ is initialized to zero and $\Psi$ is initialized accordingly via Eq. (61); the Lorentz factor $\Gamma_{\mathrm{n}}$ is then initialized via Eq. (56) and the 3-velocity $\mathbf{U}$ is initialized according to Eq. (57). We get then initial values of the Eulerian energy density $E$ and the trace of stress tensor $S$ via Eqs. (53) and (54). In these equations, we use for the proper energy density $e$ and pressure $p$ the values of the spherically symmetric initial stellar models.

\footnotetext{
${ }^{6}$ Equation (91) is valid only for equal-mass stars binaries. There also exists a more complicated formula for stars with different masses.
} 


\section{Description of one step}

At a given step, we start by determining the value of the orbital angular velocity $\Omega$ and the value of the $X$ coordinate of the rotation axis, $X_{\text {rot }}$ (see Fig. 1), by taking the gradient along $X$ of the first integral of motion (34). Demanding that the enthalpy $H$ be maximal at the center of each star (our definition of center), this results in the two equations

$$
\left.\frac{\partial}{\partial X} \ln \Gamma_{0}\right|_{\left(X_{\langle a\rangle}, 0,0\right)}=\left.\frac{\partial}{\partial X}(\nu+\ln \Gamma)\right|_{\left(X_{\langle a\rangle}, 0,0\right)} \quad a=1,2
$$

where $\ln \Gamma_{0}$ can be expressed in terms of $\Omega$ and $X_{\text {rot }}$ thanks to Eqs. (23), (24), (45) and (47):

$$
\ln \Gamma_{0}=-\frac{1}{2} \ln \left\{1-\frac{A^{2}}{N^{2}}\left[\left(\Omega Y+N^{X}\right)^{2}+\left(\Omega\left(X-X_{\text {rot }}\right)-N^{Y}\right)^{2}+\left(N^{Z}\right)^{2}\right]\right\} .
$$

Inserting this relation into Eq. (95) and setting $Y=Z=0, X=X_{\langle 1\rangle}$ or $X_{\langle 2\rangle}$ results in a system of two equations for the two unknowns $\Omega$ and $X_{\text {rot }}$. This system is solved by standard methods. Having determined $\Omega$ and $X_{\text {rot }}$, we can compute the components of the orbiting velocity $\mathbf{U}_{0}$, via Eqs. (24) and (47):

$$
U_{0}^{X}=-\frac{1}{N}\left(\Omega Y+N^{X}\right) ; \quad U_{0}^{Y}=\frac{1}{N}\left(\Omega\left(X-X_{\mathrm{rot}}\right)-N^{Y}\right) ; \quad U_{0}^{Z}=-\frac{N^{Z}}{N},
$$

where $N, N^{X}, N^{Y}$ and $N^{Z}$ are the values of the lapse function and the components of the non-rotating-coordinates shift vector taken from the previous step. From $\mathbf{U}_{0}$, we of course compute the Lorentz factor $\Gamma_{0}$ by Eq. (23). The fluid 3 -velocity with respect to the Eulerian observer, $\mathbf{U}$ is set to $\mathbf{U}_{0}$ in the synchronized case, where in the irrotational case, $\Psi$ is deduced from $\mathbf{U}_{0}$ and the previous step value of $\Psi_{0}$ via Eq. (61); the Lorentz factor $\Gamma_{\mathrm{n}}$ is then computed via Eq. (56) and the 3-velocity $\mathbf{U}$ follows from Eq. (57). The Lorentz factor $\Gamma$ between the fluid and the co-orbiting observer is deduced from the above quantities via Eq. (58).

The elliptic equation (63) for $\Psi_{0}$ is then solved by the numerical method described in Appendix B.

We then adapt the computational domains to the stars as follows. The first integral of motion (34) is written, following the splitting (80)

$$
H=H_{\langle a\rangle}^{\mathrm{c}}+\nu_{\langle a\rangle}^{\mathrm{c}}+\Phi_{\langle a\rangle, \mathrm{ext}}^{\mathrm{c}}-\nu_{\langle a\rangle}-\Phi_{\langle a\rangle, \text { ext }},
$$

where we have introduced the "external" potential

$$
\Phi_{\langle a\rangle, \mathrm{ext}}:=\nu_{\langle b \rightarrow a\rangle}-\ln \Gamma_{0}+\ln \Gamma
$$

and the superscript "c" stands for values at the center of the star. First, we rescale the auto-potential $\nu_{\langle a\rangle}$ by a factor $\alpha^{2}$ to make sure that the enthalpy vanishes at the point $\theta_{\langle a\rangle}=\pi / 2, \varphi_{\langle a\rangle}=0$ on the external boundary of domain $\mathcal{D}_{M_{\langle a\rangle}-1}^{\langle a\rangle}$ :

$$
\alpha^{2}=\frac{H_{\langle a\rangle}^{\mathrm{c}}+\Phi_{\langle a\rangle, \mathrm{ext}}^{\mathrm{c}}-\Phi_{\langle a\rangle, \mathrm{ext}}^{\mathrm{s}}}{\nu_{\langle a\rangle}^{\mathrm{s}}-\nu_{\langle a\rangle}^{\mathrm{c}}}
$$

where the superscript "s" stands for values at the point $\xi=1, \theta_{\langle a\rangle}=\pi / 2, \varphi_{\langle a\rangle}=0$ of domain $\mathcal{D}_{M_{\langle a\rangle}-1}^{\langle a\rangle}$. When the iteration converges, $\alpha$ tends to 1 . We then replace $\nu_{\langle a\rangle}$ by $\alpha^{2} \nu_{\langle a\rangle}$ in Eq. (98) to get the enthalpy field in all space. Following the technique described in Ref. [30], we then compute new functions $F_{l}(\theta, \varphi)$ and $G_{l}(\theta, \varphi)$ in the mappings (74) and (75) in order to make the outer boundary of domain $\mathcal{D}_{M_{\langle a\rangle}-1}^{\langle a\rangle}$ coincide exactly with the surface of the star. Since the collocation points of the new mapping do not coincide (in the physical space, described by the coordinates $\left.\left(r_{\langle a\rangle}, \theta_{\langle a\rangle}, \varphi_{\langle a\rangle}\right)\right)$ with that of the previous mapping, the values of the enthalpy field at the new collocation points have to be computed. The details of this computations are explained in Sect. V.A of Ref. [30]

From this new value of $H$, we compute the fluid proper baryon density $n$, proper energy density $e$ and pressure $p$ via the EOS (70). We then get the Eulerian energy density $E$ and the trace of stress tensor $S$ via Eqs. (530) and (54). These last quantities are subsequently used to evaluate the source terms of the elliptic equations (83)-(85) for the gravitational potentials. These equations are solved by means of the multi-domain scalar and vector Poisson solvers for non-compact sources described in details in Refs. [30,56]. In particular the vector Poisson equation (85) for the 
auto shift $N_{\langle a\rangle}^{i}$ is reduced to a set of 4 scalar Poisson equations according to the scheme used by Shibata and Oohara [58,10.

Before the beginning of a new step, some relaxation is performed onto the enthalpy field and the auto-potentials, according to

$$
Q^{J} \leftarrow \lambda Q^{J}+(1-\lambda) Q^{J-1}
$$

where $Q$ stands for any of the fields $H, \nu_{\langle a\rangle}, \beta_{\langle a\rangle}$ and $N_{\langle a\rangle}^{i}(a=1,2), J$ (resp. $J-1$ ) labels the current step (resp. previous step), and $\lambda$ is the relaxation factor, typically chosen to be 0.5 for $H$ and 0.65 for the auto-potentials.

For appreciably relativistic configurations, it appeared that the above relaxation is not sufficient to ensure the convergence. In this case, we update the comp-potentials not every step but every $m$ steps, with typically $m=8$. This slows the convergence but enforces it.

\section{Convergence to a given baryon mass}

In order to compute evolutionary sequences of binary neutron stars, one should be able to compute configurations for a given baryon mass, since this quantity is conserved during the gravitational-radiation driven evolution of the system. The baryon mass, given by Eq. (69), is not a natural parameter we can set in our procedure. As stated above, the freely specifiable parameters which fix one configuration are the coordinate distance $d$ between the two stellar centers and the central enthalpies $H_{\langle 1\rangle}^{\mathrm{c}}$ and $H_{\langle 2\rangle}^{\mathrm{c}}$ in each star. However, we can use the iteration procedure itself to make the final configuration have a specified baryon mass. Indeed, since the baryon mass is an increasing function of the central enthalpy (at least for the stable stars we are studying), we multiply at each step, the central enthalpy $H_{\langle 1\rangle}^{\mathrm{c}}$ by the factor

$$
\eta:=\left(\frac{2+\zeta}{2+2 \zeta}\right)^{\frac{1}{4}}
$$

where $\zeta$ is the relative discrepancy between the actual baryon mass at the considered step, $M_{\mathrm{B}}^{\langle 1\rangle J}$ and the wanted baryon mass $M_{\mathrm{B}}^{\langle 1\rangle}: \zeta:=M_{\mathrm{B}}^{\langle 1\rangle J} / M_{\mathrm{B}}^{\langle 1\rangle}-1$. When the iterative procedure converge, the factor $\eta$ tends to one. The same treatment is performed on star 2 .

\section{End of the iteration}

To control the convergence of the iterative procedure, we introduce the relative difference between the enthalpy fields of two successive steps:

$$
\delta H:=\frac{\sum_{i}\left|H^{J}\left(x_{i}\right)-H^{J-1}\left(x_{i}\right)\right|}{\sum_{i}\left|H^{J-1}\left(x_{i}\right)\right|},
$$

where the summation is extended to all the collocation points $x_{i}$ inside the star and $J$ is the step label.

We use typically $\delta H=10^{-7}$ as a criterion to end the iteration. For very high precision calculations (check with analytical solutions, see below), we use instead $\delta H=10^{-12}$.

\section{E. Treatment of cusps}

For very close configurations, an angular point (cusp) may appear at the surface of the stars, similar to that in the Roche lobe at the Lagrange point $L_{1}$ in the Roche problem. At this point the enthalpy gradient $\partial H / \partial r$ vanishes in the direction of the companion. The surface of the star is then no longer smooth and surface cannot be decribed by the differentiable mapping (74)-(75), because the functions $F_{l}(\theta, \varphi)$ and $G_{l}(\theta, \varphi)$ are assumed to be expandable in $\cos (k \theta)$ or $\sin (k \theta)$ series and in Fourier series in $\varphi$, which implies that they are smooth functions of $(\theta, \varphi)$.

The solution to this problem consists in freezing the adaptation of the mapping to the stellar surface when the enthalpy gradient becomes too small at the surface point which faces the companion star. More precisely, we define the ratio 


$$
\chi:=\frac{(\partial H / \partial r)_{\mathrm{eq}, \mathrm{comp}}}{(\partial H / \partial r)_{\mathrm{pole}}}
$$

where the index "eq, comp" stands for the value at the point $\left(\theta_{\langle a\rangle}=\pi / 2, \varphi_{\langle a\rangle}=0\right)$ on the stellar surface, whereas the "index" pole stands for the value at the point $\left(\theta_{\langle a\rangle}=0, \varphi_{\langle a\rangle}=0\right)$ on the stellar surface. When $\chi$ passes below a certain threshold $\chi_{\mathrm{fr}}$ during the iteration process, we stop the adaptation of the mapping to the surface of the star. $\chi_{\mathrm{fr}}$ is chosen typically chosen between 0.3 and 0.55 .

In this case, a Gibbs phenomenon is present. The accuracy of the calculation is then lower than when the mapping is adapted to the surface of the star. However, since the difference between the stellar surface at the domain boundary is pretty small, the Gibbs phenomenon is rather limited.

For irrotational configurations, the non-coincidence of the stellar surface with a domain boundary introduces a small error in the resolution of the equation (63) for the velocity potential $\Psi_{0}$ by means of the technique explained in Appendix B.

\section{F. Numerical implementation}

The numerical code implementing the method described above is written in the LORENE (LANGAGE OBJET POUR LA RElativité NumÉRIQuE) language [59], which is a $\mathrm{C}++$ based language for numerical relativity. A typical run makes use of 6 domains $\left(N_{\langle 1\rangle}=N_{\langle 2\rangle}=3\right.$ and $\left.M_{\langle 1\rangle}=M_{\langle 2\rangle}=1\right)$, with $N_{r} \times N_{\theta} \times N_{\varphi}=33 \times 21 \times 20$ coefficients in each domain. The corresponding memory requirement is $232 \mathrm{MB}$ for an irrotational configuration. A computation involves $\sim 250$ steps, which takes $14 \mathrm{~h}$ on one CPU of a SGI Origin200 computer (MIPS R10000 processor at 180 $\mathrm{MHz}$ ). If the number of coefficients is lowered to $N_{r} \times N_{\theta} \times N_{\varphi}=25 \times 17 \times 16$, the memory requirement and CPU times becomes respectively $100 \mathrm{MB}$ and $6 \mathrm{~h} 30 \mathrm{~min}$.

Note that due to the rather small memory requirement, runs can be performed in parallel on a multi-processor platform. This is especially useful to compute sequences of configurations.

Both Newtonian and relativistic configurations, either corotating or irrotational, are calculated by the same code. Only the parts of the computation specific to one of these four cases are treated by different branches of the code.

\section{TESTS OF THE NUMERICAL CODE}

After constructing a numerical code for calculation of binary neutron stars, we must assert its validity by performing self-consistency checks and comparing the results with those of analytic solutions or those of previous numerical works. The plan of the tests of the numerical code is as follows. For the irrotational configurations:

1. Check the convergence of the iterative procedure.

2. Check the convergence of the global quantities when increasing the number of coefficients of the spectral method.

3. Check the decay of the relative error in the virial theorem for Newtonian binary systems when increasing the number of coefficients of the spectral method.

4. Check the agreement with some analytic solutions for Newtonian binary systems.

5. Check the agreement with previous numerical solutions for Newtonian binary systems.

6. Check the coincidence of the results of the purely Newtonian calculation with those of general relativistic one with small compactness.

and for the corotating configurations:

1. Check the agreement with previous numerical solutions of relativistic binary systems.

For the purpose of the test computations, we consider identical star binary systems with the polytropic equation of state

$$
\begin{aligned}
& n(H)=\left[\frac{\gamma-1}{\gamma} \frac{m_{\mathrm{B}}}{\kappa}(\exp (H)-1)\right]^{1 /(\gamma-1)} \\
& p(H)=\kappa n(H)^{\gamma} \\
& e(H)=\frac{\kappa}{\gamma-1} n(H)^{\gamma}+m_{\mathrm{B}} n(H),
\end{aligned}
$$

where $\gamma, \kappa$ and $m_{\mathrm{B}}$ are some constants. For $m_{\mathrm{B}}$ we will use $m_{\mathrm{B}}=1.66 \times 10^{-27} \mathrm{~kg}$ (mean baryon mass). 


\section{A. Self-consistency checks}

First of all, we show in Fig. 3 the convergence of the iterative procedure described in Sect. IV D. This convergence is measured by means of the relative difference $\delta H$ between two successive steps values of the enthalpy field, as given by Eq. (103). The bump around the 70th step corresponds to the switch on the procedure of convergence towards a given baryon mass, as described in Sect. IVD3. One can notice systematic oscillations in the convergence curve every 8 steps. They result from the fact that the comp-potentials are updated only every 8 steps, as discussed in Sect. IVD 2. We stop the iterations when the convergence has reached the $\delta H=10^{-7}$ level (dashed horizontal line in Fig. 3).

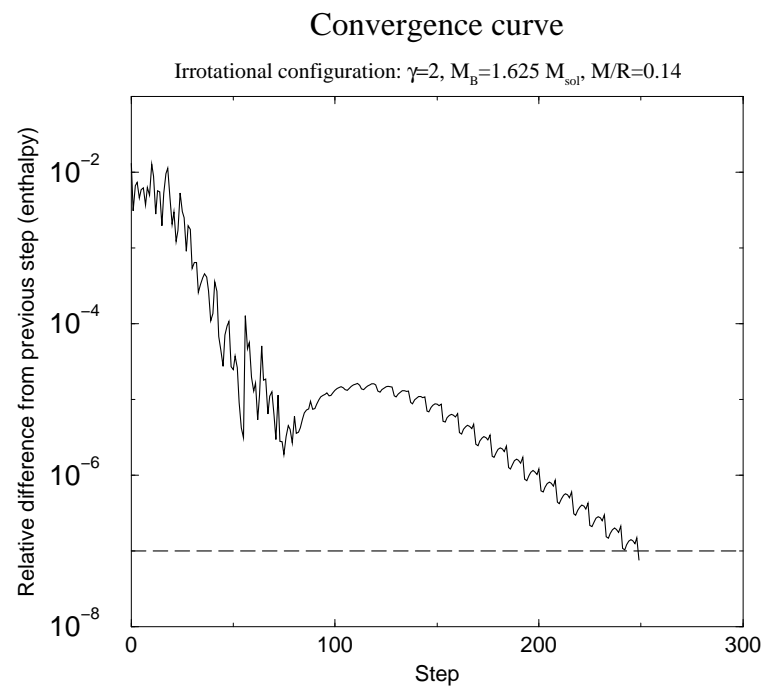

FIG. 3. Convergence (measured by the relative difference $\delta H$ in the enthalpy field between two successive steps) of the iterative procedure for one of the irrotational models with $N_{r} \times N_{\theta} \times N_{\varphi}=33 \times 21 \times 20$ collocation points. The bump around the 70th step corresponds to the switch on of the procedure of convergence towards a given baryon mass.

Next, we show the convergences of the global quantities (i.e. ADM mass and total angular momentum) for one configuration when the number of spectral coefficients (or equivalently the number of collocation points, cf. Sect. IV B) is increased. Furthermore, we present the convergence of the relative change in central energy density along a sequence when we increase the number of spectral coefficients. The calculations are performed for the case of $\gamma=2$, $\kappa=0.0332 \rho_{\text {nuc }}^{-1} c^{2}\left(\rho_{\text {nuc }}:=1.66 \times 10^{17} \mathrm{~kg} \mathrm{~m}^{-3}\right)$; the baryon mass is $M_{B}=1.625 M_{\odot}$, which corresponds to the compactness $M / R=0.14$ for isolated spherical stars. The coordinate separation $d$ is taken to be $60 \mathrm{~km}$. Six domains are used, with the following parameters (using the notations of Sect. IV A): $N_{\langle 1\rangle}=N_{\langle 2\rangle}=3, M_{\langle 1\rangle}=M_{\langle 2\rangle}=1$, with the same number of coefficients in each domains: $N_{r}^{\langle 1\rangle}(0)=N_{r}^{\langle 1\rangle}(1)=\cdots=: N_{r}, N_{\theta}^{\langle 1\rangle}(0)=N_{\theta}^{\langle 1\rangle}(1)=\cdots=: N_{\theta}$ and $N_{\varphi}^{\langle 1\rangle}(0)=N_{\varphi}^{\langle 1\rangle}(1)=\cdots=: N_{\varphi}$.

The ADM mass and total angular momentum are shown in Figs. 1 and 5 as functions of $N_{r}$. We used the following numbers of spectral coefficients: $N_{r} \times N_{\theta} \times N_{\varphi}=9 \times 7 \times 6,13 \times 9 \times 8,17 \times 13 \times 12,25 \times 17 \times 16,33 \times 21 \times 20$ and $41 \times 25 \times 24$. In Fig. 6, we give the relative change in central energy density along a quasiequilibrium sequence for various numbers of spectral coefficients: $\left(N_{r}, N_{\theta}, N_{\varphi}\right)=(9,7,6),(13,9,8),(17,13,12),(25,17,16),(33,21,20)$, and $(33,25,24)$. We find that these global quantities settle to a constant value (variations below $\sim 10^{-5}$ ) for $N_{r} \geq 25$. 


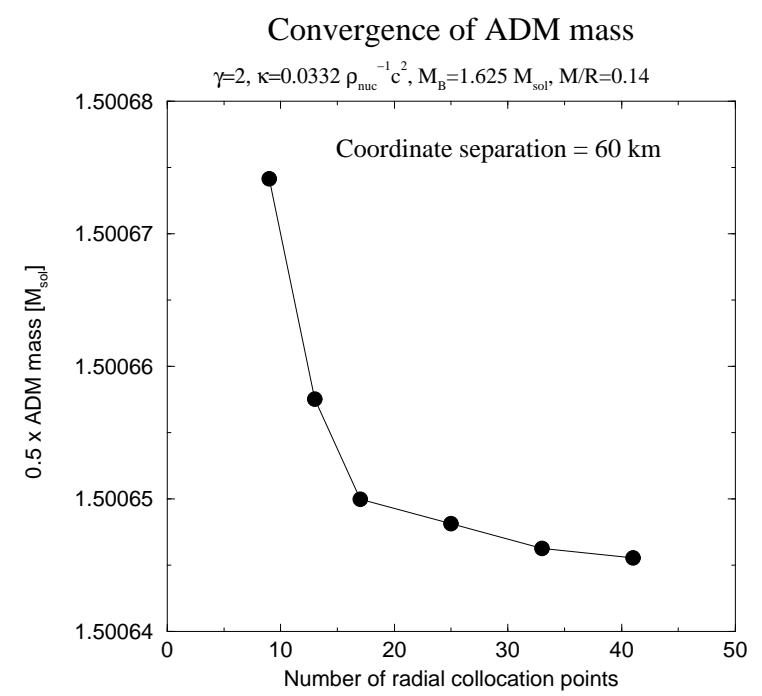

FIG. 4. Convergence of the ADM mass for one of the irrotational relativistic models, as the number of collocation points (or equivalently of spectral coefficients) is increased.

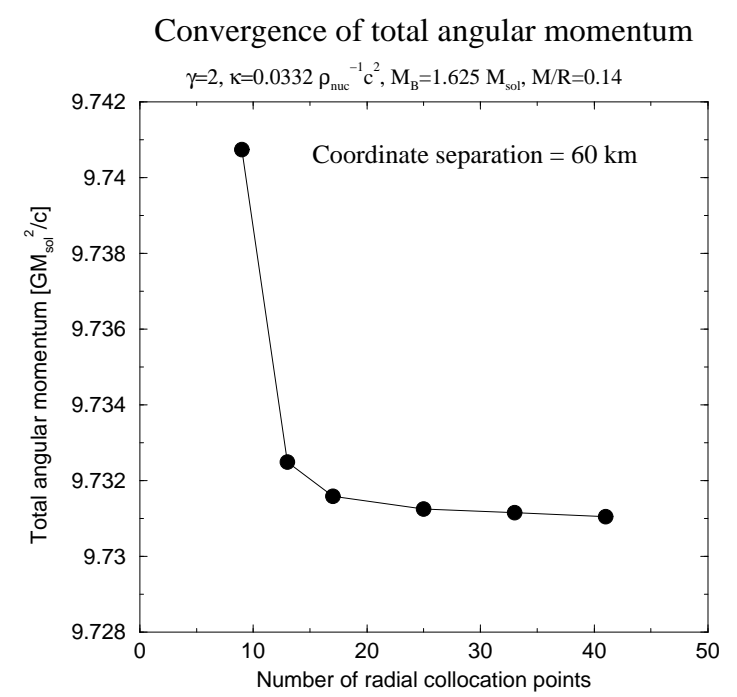

FIG. 5. Same as Fig. 1 but for the total angular momentum. 


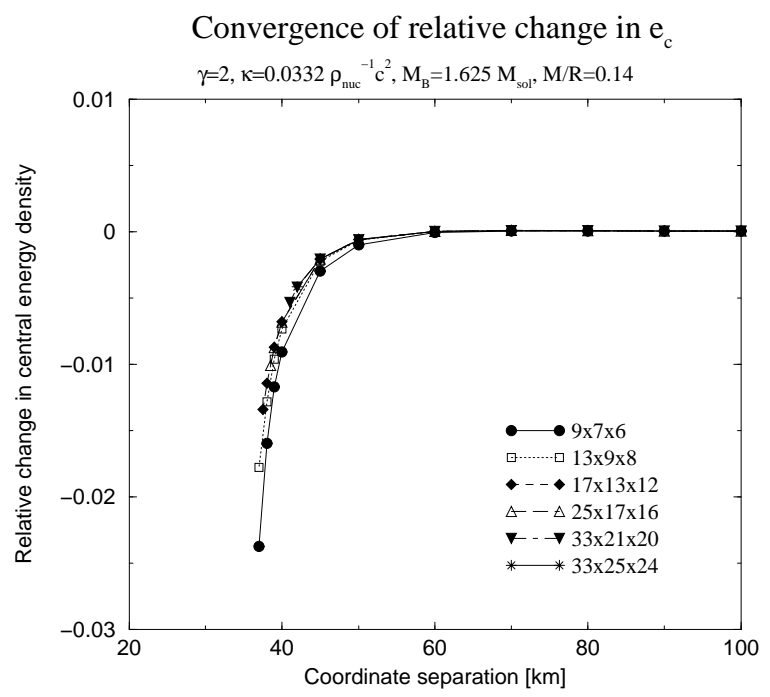

FIG. 6. Convergence of the evolution of the central energy density along a quasiequilibrium sequence, as the number $N_{r} \times N_{\theta} \times N_{\varphi}$ of collocation points (or equivalently of spectral coefficients) is increased.

\section{B. Tests in the Newtonian regime}

In order to test Newtonian calculations, we compute a $M_{B}=10^{-3} M_{\odot}$ Newtonian sequence based on a polytropic equation of state with $\gamma=2$ and $\kappa=0.0332 \rho_{\text {nuc }}^{-1} c^{2}$. In this case, the central baryon density and the radius of infinitely separated stars becomes $1.081 \times 10^{-3} \rho_{\text {nuc }}$ and $20.57 \mathrm{~km}$, respectively. Note that the Newtonian limit of the polytropic equation of state (105)-(107) is obtained for $H \ll 1$ and reads

$$
\begin{aligned}
n(H) & =\left[\frac{\gamma-1}{\gamma} \frac{m_{\mathrm{B}}}{\kappa} H\right]^{1 /(\gamma-1)} \\
p(H) & =\kappa n(H)^{\gamma} \\
e(H) & =m_{\mathrm{B}} n(H) .
\end{aligned}
$$

\section{Virial theorem}

A useful method to estimate the global numerical error in Newtonian computations is to calculate the relative error in the virial theorem. This latter states that $2 T+W+3 P=0$, where $T, W$, and $P$ denote respectively the kinetic energy of the binary system, its gravitational potential energy and the volume integral of the fluid pressure. We therefore define the virial error as

$$
\text { Error }=\frac{|2 T+W+3 P|}{|W|}
$$




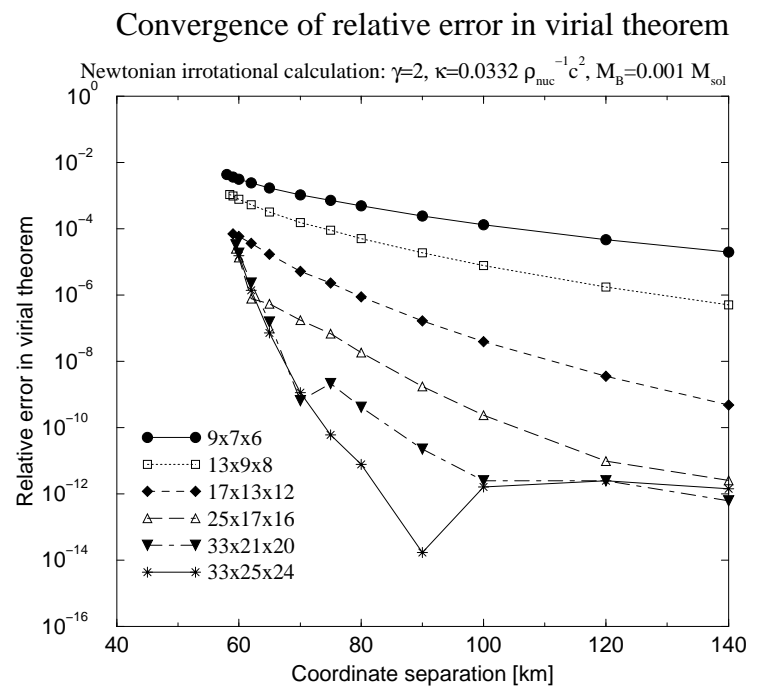

FIG. 7. Relative error in the virial theorem along an evolutionary sequence, for various numbers $N_{r} \times N_{\theta} \times N_{\varphi}$ of collocation points (or equivalently of spectral coefficients).

This error estimator is shown along a constant baryon number sequence in Fig. 7. In order to check the convergence of the numerical method, we present various cases of increasing number of spectral coefficients: $N_{r} \times N_{\theta} \times N_{\varphi}=9 \times 7 \times 6$, $13 \times 9 \times 8,17 \times 13 \times 12,25 \times 17 \times 16,33 \times 21 \times 20$ and $33 \times 25 \times 24$. We used $\delta H=10^{-12}$ as the criterion to end the iteration. It is found from Fig. 7 that for large separations the relative error converges to $10^{-12}$ when the number of spectral coefficients is increased, which is of the order of $\delta H$. Anyway, one cannot go much further, even if $\delta H$ is lowered significantly, because of the use of 15 digits numbers (double precision) and the resulting accumulation of round-off errors in the arithmetical operations. Besides, we notice in Fig. If the appearance of a rapidly increasing error for closer separations. Note however that at the point of closest approach (cusp point) this error is below $10^{-4}$ (except for the low numbers of spectral coefficients), which is very satisfactory. Finally, we see on Fig. 7 that when we increase the number of polar and azimuthal collocation points fixing the number of radial ones, the relative error in the virial theorem becomes better around intermediate separations.

\section{Comparison with analytic solutions}

Until recently the only analytic solutions for binary stars were constructed with incompressible fluid and belong to the so-called families of Roche-Riemann or Darwin-Riemann ellipsoids ${ }^{7}$ 60.61] (see Ref. 62 for a good introduction to these ellipsoidal solutions). We have presented elsewhere [30] the comparison with Roche ellipsoids (the sub-class of Roche-Riemann ellipsoids constituted by synchronized systems), as a validation of our multi-domain spectral approach with surface-fitted coordinates. As can be seen from Fig. 6 of Ref. [30] the numerical error is decreasing exponentially with the number of spectral coefficients (the so-called evanescent error typical of spectral methods), reaching $10^{-9}$ for a Roche ellipsoid with axis ratios $a_{2} / a_{1}=0.75$ and $a_{3} / a_{1}=0.68$.

The case of compressible fluid bodies has been investigated recently by Taniguchi \& Nakamura [63,64], who have obtained semi-analytic solutions for equilibrium sequences of irrotational binary polytropic stars in Newtonian gravity. For an equal-mass star binary system with $\gamma=2$, they have produced the following simple equations for the total energy $E$, the total angular momentum $J$, the orbital angular velocity $\Omega$ and the relative change in central baryon density:

$$
\begin{aligned}
& E=\frac{G M^{2}}{R_{0}}\left[-1-\frac{1}{2}\left(\frac{R_{0}}{d}\right)+2\left(\frac{15}{\pi^{2}}-1\right)\left(\frac{R_{0}}{d}\right)^{6}\right] \\
& J=\frac{1}{2} M d^{2} \Omega\left[1+\text { higher term than } O\left(\left(\frac{R_{0}}{d}\right)^{6}\right)\right],
\end{aligned}
$$

\footnotetext{
${ }^{7}$ Note however that these solutions are not exact for the gravitational potential of the companion must be truncated to the second order to get perfectelly ellipsoidal shapes
} 


$$
\begin{aligned}
& \Omega^{2}=\frac{2 G M}{d^{3}}\left[1+6\left(\frac{15}{\pi^{2}}-1\right)\left(\frac{R_{0}}{d}\right)^{5}\right], \\
& \delta \rho_{c}=\frac{\rho_{c}-\rho_{c 0}}{\rho_{c 0}}=-\frac{45}{2 \pi^{2}}\left(\frac{R_{0}}{d}\right)^{6},
\end{aligned}
$$

where $d$ is the separation, $\rho_{c}$ the central density, $R_{0}$ the radius of the spherical star of mass $M$ (i.e. the radius at infinite separation) and $\rho_{c 0}$ the central density of this spherical star. These equations are exact up to $O\left(\left(R_{0} / d\right)^{6}\right)$ and are very valuable to check the validity of the Newtonian limit of our code, in particular the solution of equation (63) for the velocity potential. Indeed the Darwin-Riemann solutions could not have been used for testing this important part of the code because Eq. (63) is degenerate for an incompressible fluid $(\zeta=\infty)$.

First, we compare our numerical results with Taniguchi \& Nakamura's analytic solutions for global quantities (such that total energy, total angular momentum, orbital angular velocity, and relative change in the central baryon density) along an evolutionary sequence in Figs. 8- 11. For the numerical computation, we use $N_{r} \times N_{\theta} \times N_{\varphi}=33 \times 25 \times 24$ spectral coefficients in each domain and the criterion $\delta H=10^{-12}$ to end the iterations. It is found from these figures that the numerical results agree very well with the analytic ones. Note that the analytic solution ends at the contact point, whereas the numerical one ends before (when a cusp appears at the stellar surface, cf. Sect. IVE). However the analytic solution, based on an expansion up to $O\left(\left(R_{0} / d\right)^{6}\right)$, loses its accuracy for very close separations and cannot be used to test the code in this regime.

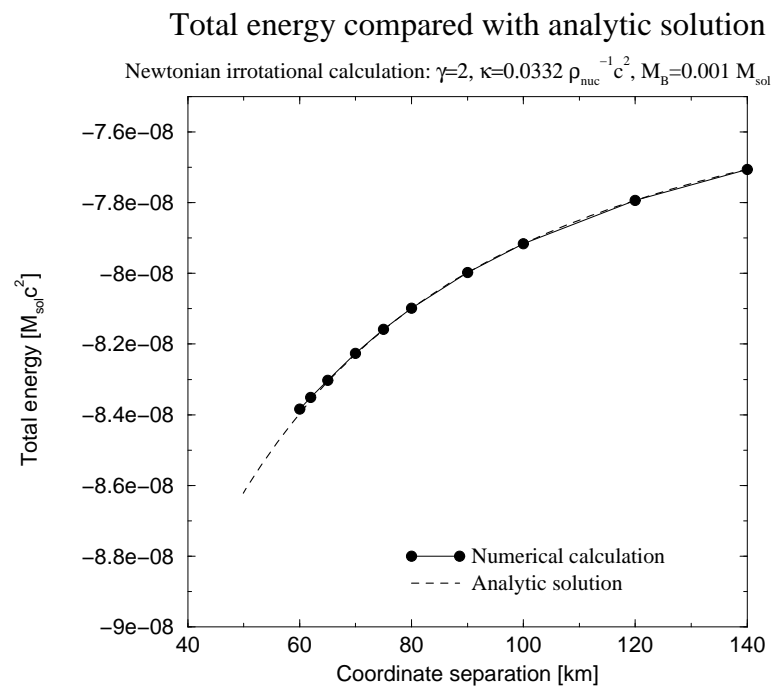

FIG. 8. Total energy compared with Taniguchi \& Nakamura's analytic solution 63,64 along an evolutionary sequence. Solid and dashed lines denote the results of numerical and analytic calculations, respectively.

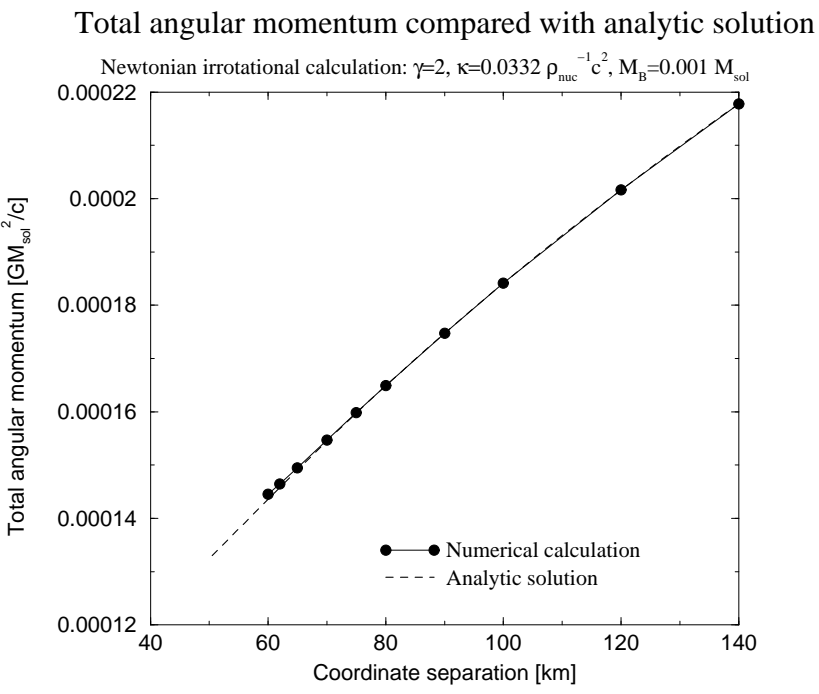


FIG. 9. Same as Fig. 8 but the total angular momentum.

Orbital angular velocity compared with analytic solution

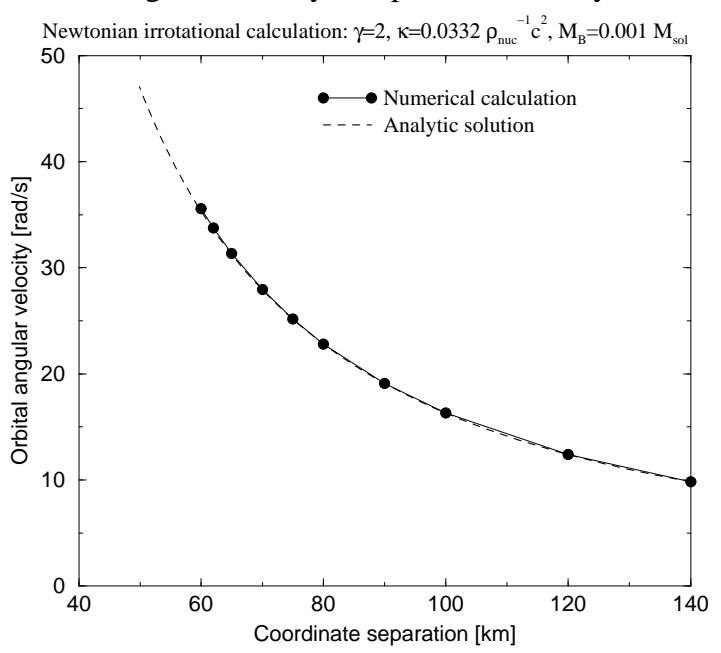

FIG. 10. Same as Fig. 8 but the orbital angular velocity.

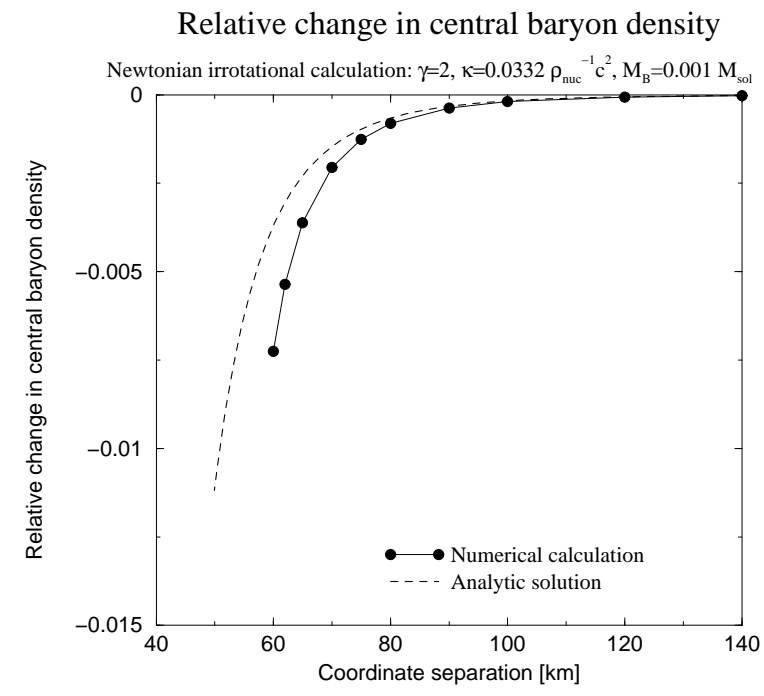

FIG. 11. Same as Fig. 8 but the relative change in central baryon density.

In order to investigate the discrepancy between the results from the numerical code and those from Taniguchi \& Nakamura's analytic solution, we present the relative differences on global quantities as functions of the separation in a log-log plot in Fig. 12. The relative differences are defined as follows:

$$
\begin{aligned}
& \frac{E_{\text {num }}-E_{\text {ana }}}{G M^{2} / R_{0}}, \\
& \frac{J_{\text {num }}-J_{\text {ana }}}{M d^{2} \Omega_{\text {Kep }} / 2} \\
& \frac{\Omega_{\text {num }}-\Omega_{\text {ana }}}{\Omega_{\text {Kep }}} \\
& \left|\delta \rho_{c: \text { num }}-\delta \rho_{c: \text { ana }}\right|
\end{aligned}
$$

where $\Omega_{\text {Kep }}$ is the Keplerian velocity for point mass particles: 


$$
\Omega_{\mathrm{Kep}}:=\left(\frac{2 G M}{d^{3}}\right)^{1 / 2} .
$$

Two reference lines, proportional to $\left(d / R_{0}\right)^{-9}$ and $\left(d / R_{0}\right)^{-7.5}$, have been drawn in Fig. 12 in order to check the slope of the results easily.

It is found that for separations closer than $d / R_{0}=10$, the discrepancies between numerical and analytic solutions for the energy $E$ and the relative change in central density $\delta \rho_{c}$ are both proportional to $\sim\left(d / R_{0}\right)^{-9}$, and become $\sim\left(d / R_{0}\right)^{-13}$ around $d / R_{0} \sim 3$. At first glance, this agreement between the numerical and analytical solutions seems too good, because we know that the next order term missing in Eqs. (112) and (115) is $O\left(\sim\left(d / R_{0}\right)^{-8}\right)$. We interpret the fact that this term does not show up in Fig. 12 by the fact that it is produced by the octupole deformation, which should be very small. Of course, for separations much larger than $d / R_{0}=10$, the term proportional to $\sim\left(d / R_{0}\right)^{-8}$ would dominate the inclination of the lines.

For the residual terms of the angular momentum $J$ and the orbital angular velocity $\Omega$, we can see that while they are proportional to $\sim\left(d / R_{0}\right)^{-7}$ around $d / R_{0} \sim 10$, the term proportional to $\sim\left(d / R_{0}\right)^{-8}$ dominates for separations closer than $d / R_{0} \sim 6$, and finally goes up to $\sim\left(d / R_{0}\right)^{-12}$. We can explain this dependence as follows. First, the high order expansion of $\Omega$ can be written as

$$
\Omega=\Omega_{\mathrm{Kep}}\left[1+O\left(\left(\frac{d}{R_{0}}\right)^{-5}\right)+O\left(\left(\frac{d}{R_{0}}\right)^{-7}\right)+O\left(\left(\frac{d}{R_{0}}\right)^{-8}\right)+\cdots\right] .
$$

Note here that the second term inside the brackets comes from the quadrupole deformation of the star and is included in the analytic solution [Eq. (114)]. After subtracting the analytic solution (114) from expression (121), there remains the term $O\left(\left(d / R_{0}\right)^{-7}\right)$ as a leading one. Therefore it dominates the behavior of the curve of the relative difference in $\Omega$ around $d / R_{0} \sim 10$.

On the other hand, the angular momentum is expandable as

$$
J=\frac{M}{2} d^{2} \Omega\left[1+O\left(\left(\frac{d}{R_{0}}\right)^{-8}\right)+\cdots\right] .
$$

This means that after subtracting the analytic solution (113) and normalizing by $M d^{2} \Omega_{\mathrm{Kep}} / 2$, the leading term of $J$ comes from $\Omega$, because this latter has a term proportional to $\left(d / R_{0}\right)^{-7}$. This explains why the discrepancy curves for $J$ and $\Omega$ have almost the same behavior.

From the above discussion about the slopes of Fig. 12 curves, we can conclude that the numerical solution agrees with the semi-analytical one within the accuracy of this latter, i.e. the increase of the discrepancy when the separation decreases is due to missing (high order) terms in the analytic solution (112)-(115). Finally, we see from Fig. 12 that even if the baryon mass is changed by a factor larger than $10^{3}$, the numerical and analytical solutions agree in the very same manner.

Relative difference from analytic solution

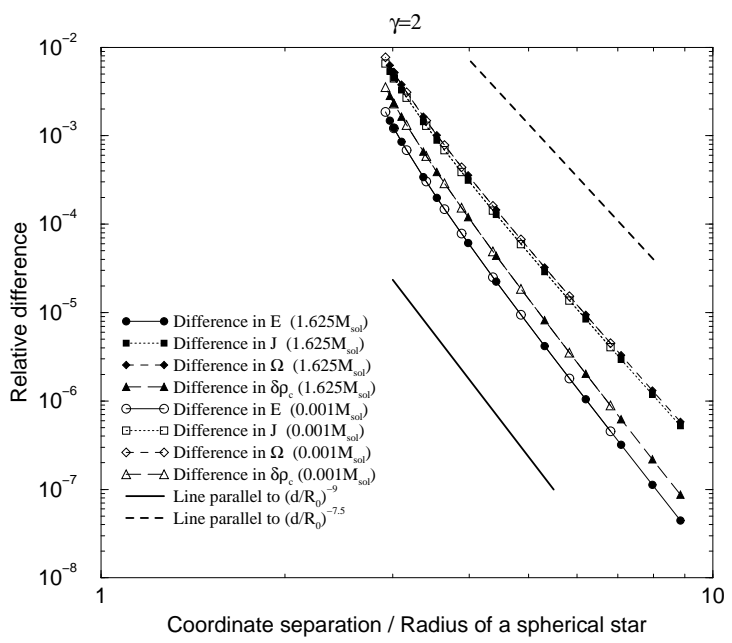

FIG. 12. Relative differences in total energy $E$, total angular momentum $J$, orbital angular velocity $\Omega$, and relative change in central baryon density $\delta \rho_{\mathrm{c}}$ when comparing the numerical solution with Taniguchi \& Nakamura's analytic solution [63.64] along an equilibrium sequence. The horizontal axis denotes logarithmically $d / R_{0}$, where $d$ is the separation between the two stellar centers and $R_{0}$ the stellar radius at infinite separation. The thick solid and thick dashed lines are reference ones in order to check the inclinations of the results easily. 
Next, we compare the internal velocity field in the co-orbiting frame with that of Taniguchi \& Nakamura's analytic solution. We focus on the velocity component along the orbital axis ( $z$-axis), because it is three orders of magnitude smaller than the $x$ and $y$-axis components even for the case of closer separation, and is therefore a very valuable quantity to check whether the equation of continuity (63) is well solved or not. In Figs. 13 - 15, we show the velocity $z$-component as a function of the radial distance $r_{\langle 1\rangle}$ from the center of star 1 along three directions: $\left(\theta_{\langle 1\rangle}, \varphi_{\langle 1\rangle}\right)=$ $(\pi / 4, \pi / 4),(\pi / 4, \pi / 2)$, and $(\pi / 4,3 \pi / 4)$ and for the orbital separations $d=200 \mathrm{~km}, 140 \mathrm{~km}, 100 \mathrm{~km}$, and $70 \mathrm{~km}$. It is found that the numerical results agree nicely with those of analytic calculations. Once again, note that the discrepancy at small separation comes from the fact that the analytic solution deviates substantially from the exact solution.

\section{Z-axis component of internal velocity field}

$(\theta, \varphi)=(\pi / 4, \pi / 4)$
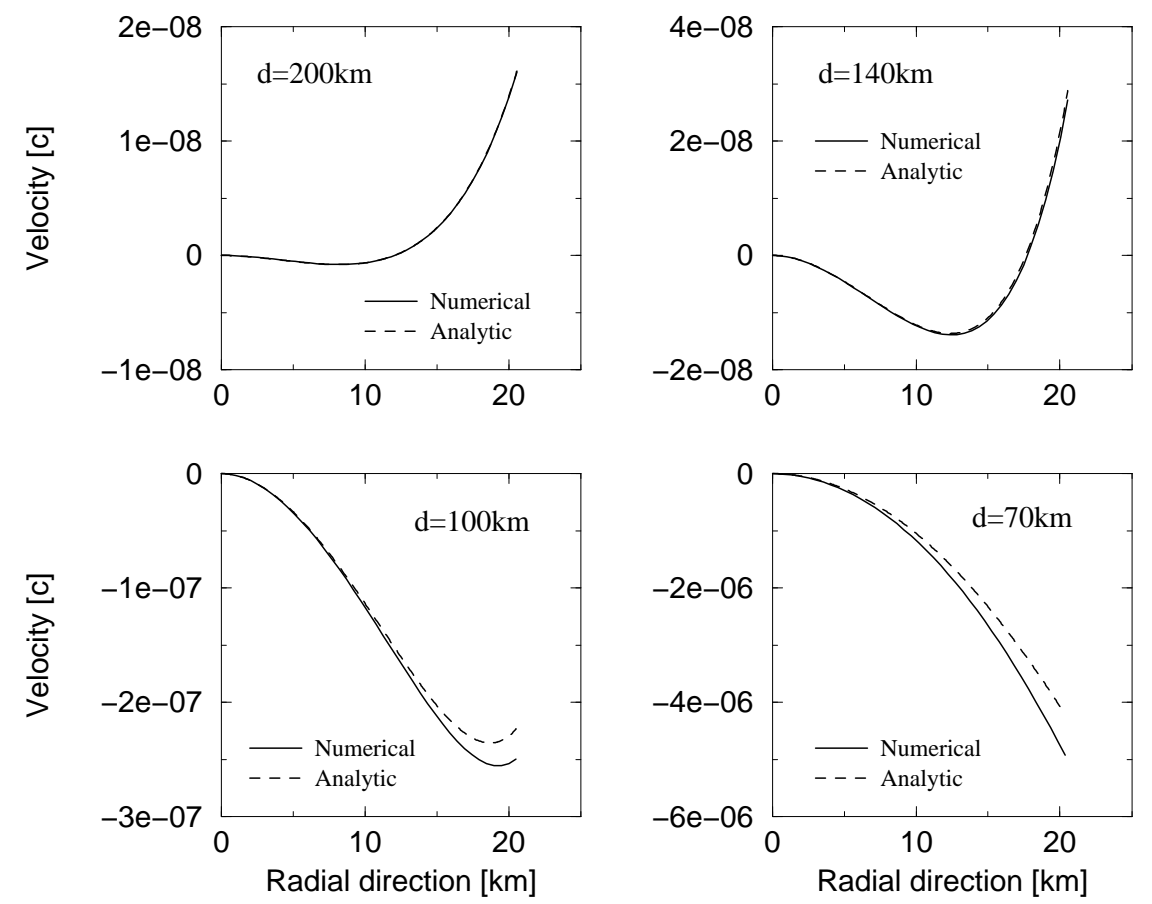

FIG. 13. The $z$-axis component of the internal velocity field in the co-orbiting frame compared with Taniguchi \& Nakamura's analytic solution [63]64]. The horizontal line denotes the radial distance from the center to the surface of star 1 , in the direction $\left(\theta_{\langle 1\rangle}, \varphi_{\langle 1\rangle}\right)=(\pi / 4, \pi / 4)$. The four panels are snapshots at different separations: $200 \mathrm{~km}, 140 \mathrm{~km}, 100 \mathrm{~km}$, and $70 \mathrm{~km}$. Solid and dashed lines denote the results of numerical and analytic calculations, respectively. 
Z-axis component of internal velocity field

$(\theta, \varphi)=(\pi / 4, \pi / 2)$
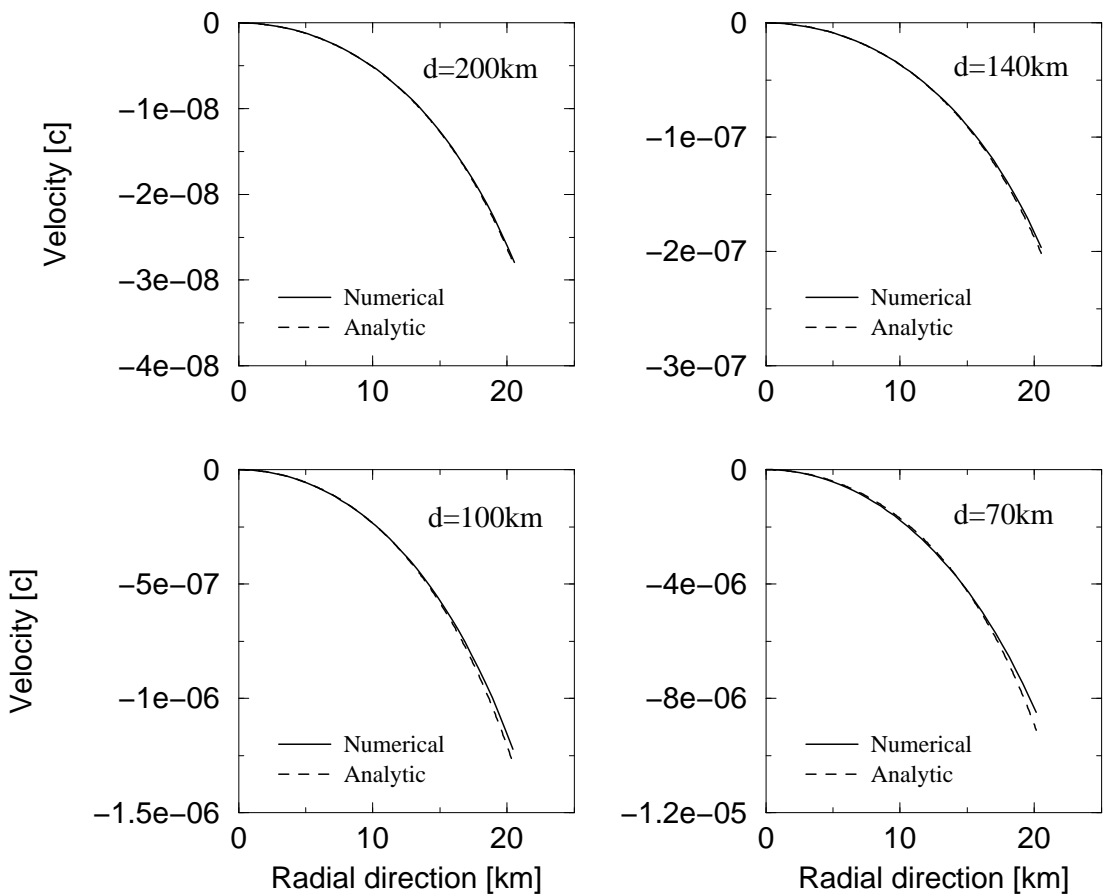

FIG. 14. Same as Fig. 13 but for the direction $\left(\theta_{\langle 1\rangle}, \varphi_{\langle 1\rangle}\right)=(\pi / 4, \pi / 2)$.

Z-axis component of internal velocity field

$(\theta, \varphi)=(\pi / 4,3 \pi / 4)$
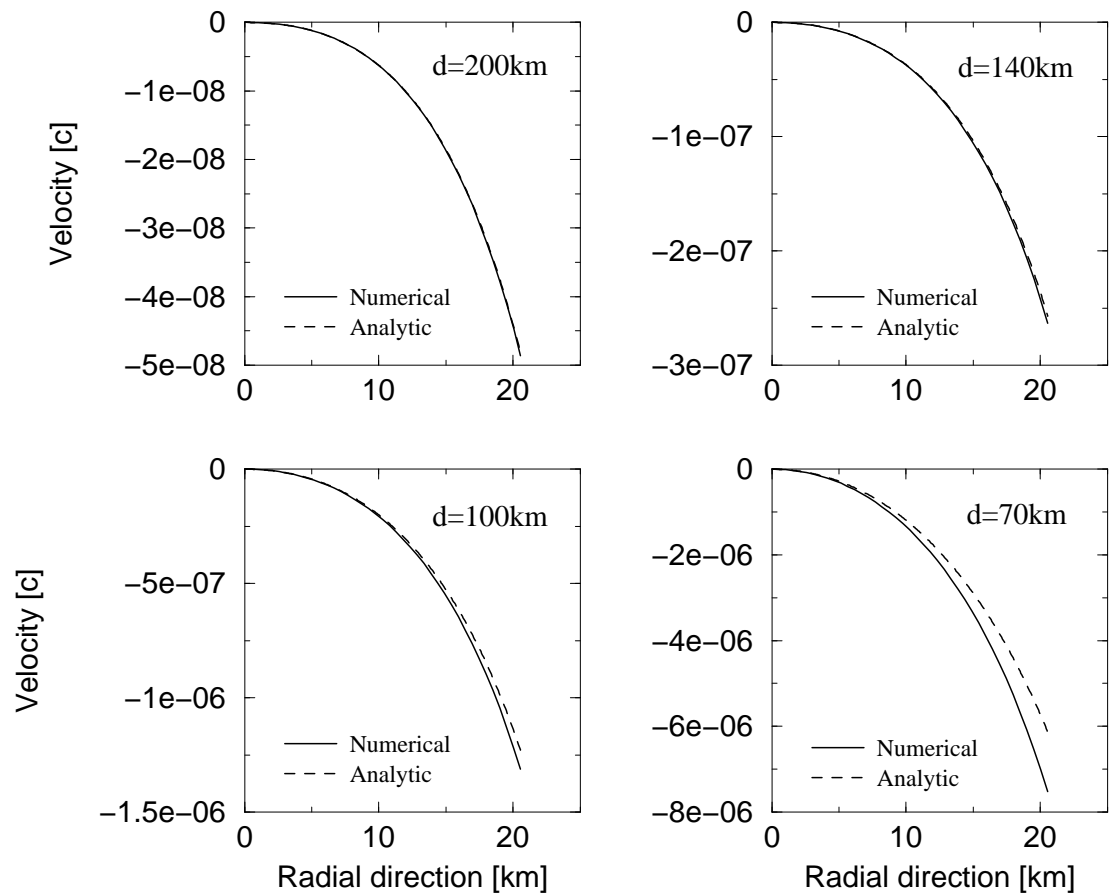

FIG. 15. Same as Fig. 13 but for the direction $\left(\theta_{\langle 1\rangle}, \varphi_{\langle 1\rangle}\right)=(\pi / 4,3 \pi / 4)$. 


\section{Comparison with previous numerical solutions}

As a final test for Newtonian computations, we compare our results with those of Uryu \& Eriguchi [43] for polytropic equation of state with $\gamma=5 / 3,2$ and 3, corresponding to polytropic indices $n=1.5,1$ and 0.5 respectively $(\gamma=$ $1+1 / n)$. The comparison is presented in Table [], where the upper lines for each configuration are the results of Uryu \& Eriguchi and the lower ones are ours. We have chosen the configurations $\tilde{d}=3.6$ in Tables 2 , 4, and 5 of Uryu \& Eriguchi [43]. Here, our results are calculated by using $N_{r} \times N_{\theta} \times N_{\varphi}=33 \times 25 \times 24$ spectral coefficients in each of the 6 domains and we adopted the same definitions as in Uryu \& Eriguchi's article 443, namely

$$
\begin{aligned}
\bar{d}_{G} & :=\frac{d_{G}}{R_{0}}, \\
\bar{\Omega} & :=\frac{\Omega}{\left(\pi G \bar{\rho}_{0}\right)^{1 / 2}}, \\
\bar{J} & :=\frac{J}{\left(G M^{3} R_{0}\right)^{1 / 2}}, \\
\bar{E} & :=\frac{E}{G M^{2} / R_{0}},
\end{aligned}
$$

where $d_{G}$ is the distance between the two stellar centers of mass and $\bar{\rho}_{0}:=M /\left(4 \pi R_{0}^{3} / 3\right)$.

One can see from Table i that our results coincide with those of Uryu \& Eriguchi within $0.3 \%$ for physical values such as the total energy, the total angular momentum and the orbital angular velocity. Note that the label $\tilde{d}$ of Uryu \& Eriguchi [43] configurations is the orbital separation between the geometrical centers of two stars normalized by the geometrical radius of the star along the $x$-axis. In our computation, since the geometrical separation is obtained after calculation, we cannot fix $\tilde{d}$ initially. Therefore we use the corresponding separation between the centers of mass of two stars which Uryu \& Eriguchi gave in their paper [43] as the orbital separation between the centers of two stars $\bar{d}=d / R_{0}$. Although our definition of the center of the star, which is the location of the maximum enthalpy

\begin{tabular}{|c|c|c|c|}
\hline Separation & $\bar{\Omega}$ & $\bar{J}$ & $\bar{E}$ \\
\hline \multicolumn{4}{|c|}{$\gamma=3(n=0.5)$} \\
\hline$\overline{d_{G}}=3.804$ & 0.2219 & 1.385 & -1.241 \\
\hline $\bar{d}=3.804$ & 0.2211 & 1.385 & -1.242 \\
\hline \multicolumn{4}{|c|}{$\gamma=2(n=1)$} \\
\hline$\overline{d_{G}}=3.753$ & 0.2259 & 1.371 & -1.133 \\
\hline $\bar{d}=3.753$ & 0.2252 & 1.373 & -1.133 \\
\hline \multicolumn{4}{|c|}{$\gamma=5 / 3(n=1.5)$} \\
\hline$\overline{d_{G}}=3.726$ & 0.2279 & 1.364 & -0.9921 \\
\hline $\bar{d}=3.726$ & 0.2274 & 1.367 & -0.9911 \\
\hline
\end{tabular}
(Sect. IV A ), is different from the center of mass, the relative difference between these centers is only about $0.01 \%$ around $\tilde{d}=3.6$.

TABLE I. Comparison with the results of Uryu \& Eriguchi (1998). 


\section{Test of the Newtonian limit of relativistic calculations}

We have made many tests of the code in the Newtonian regime up to now, so that we are rather confident in the accuracy of the Newtonian part of the code. As a next step, we compare the results of relativistic calculations with small compactness $\left(M / R=7.18 \times 10^{-5}\right)$ with those of Newtonian ones. In Figs. 16 - 18, the total energy, the total angular momentum and the orbital angular velocity are shown along a sequence. We use $N_{r} \times N_{\theta} \times N_{\varphi}=$ $25 \times 17 \times 16$ spectral coefficients in each domain. It appears clearly that the results of the small compactness relativistic computation coincide with those of the Newtonian computation, as it should be.

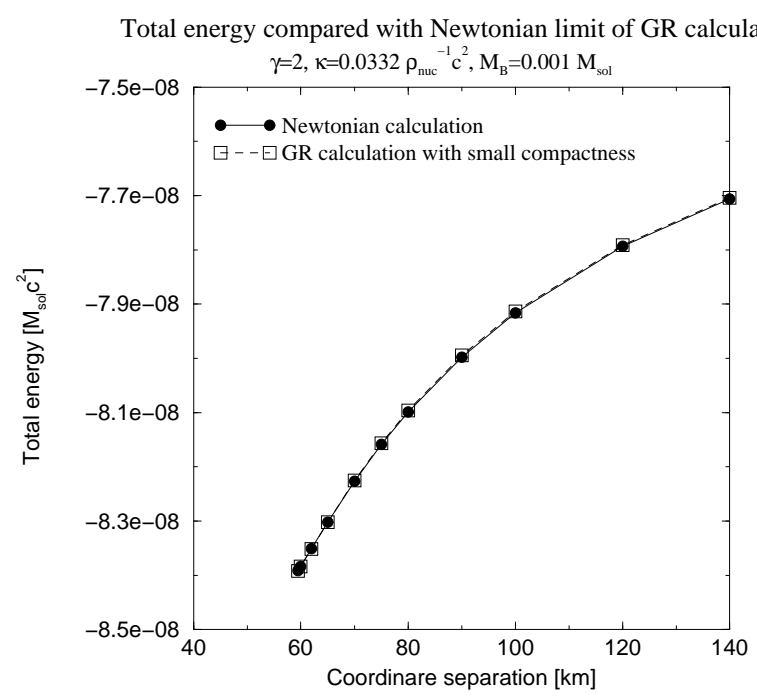

FIG. 16. Total energy of a relativistic sequence of small compactness $\left(M / R=7.18 \times 10^{-5}\right)$ compared with that of that of a Newtonian sequence of the same mass. Solid line with filled circles denotes the Newtonian computation and dashed line with squares denotes the relativistic one.

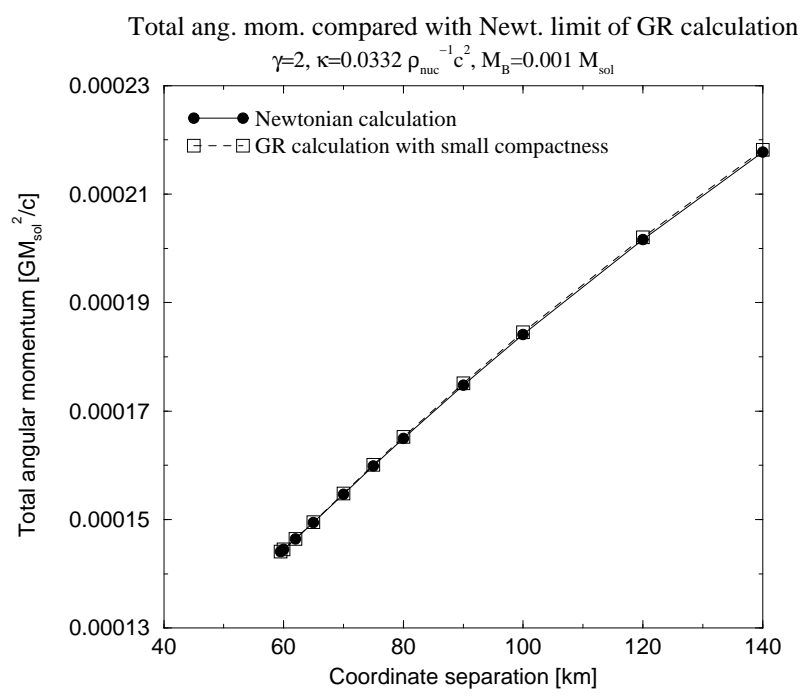

FIG. 17. Same as Fig. 16 but for the total angular momentum. 


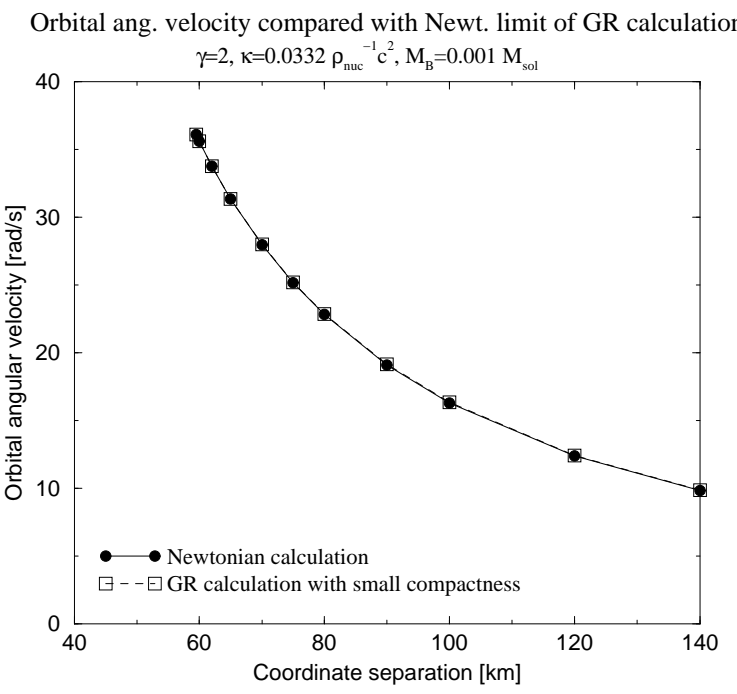

FIG. 18. Same as Fig. 16 but for the orbital angular velocity.

\section{Comparison with previous relativistic numerical solutions}

\section{Corotating case}

As a check of for relativistic computations, we compare our results for corotating configurations with those of Baumgarte et al. [26]. We have chosen Tables III and VI of their paper, and compare the results of two different separations $z_{A}=0.2$ and 0.3 in each table. We use $N_{r} \times N_{\theta} \times N_{\varphi}=33 \times 25 \times 24$ spectral coefficients in each domain and the criterion $\delta H=10^{-7}$ to end the computation of one configuration. We adopt the same equation of state (polytropic with $\gamma=2$ ), the same value of the separation $r_{C}=d / 2$ and the same value of the baryon mass $\bar{M}_{0}$. These results are shown in Table [1] where the upper lines for each configuration denote the results of Baumgarte et al., and the lower ones correspond to our results. We find a relative discrepancy of $2 \%$ on $\bar{\Omega}, 4.5 \%$ on $q^{\max }, 0.07 \%$ on $\bar{M}$, $0.6 \%$ on $\bar{J}, 4.5 \%$ on $\bar{r}_{A}$, and $1.5 \%$ on $\bar{r}_{B}$.

TABLE II. Comparison with the results of Baumgarte et al. (1998)

\begin{tabular}{|c|c|c|c|c|c|c|c|c|}
\hline$z_{A}$ & $\bar{M}_{0}$ & $q^{\max }$ & $\bar{M}$ & $\bar{J}$ & $\bar{\Omega}$ & $\bar{r}_{A}$ & $\bar{r}_{C}$ & $\bar{r}_{B}$ \\
\hline \multicolumn{9}{|c|}{ TABLE III $(M / R=0.05)$} \\
\hline \multirow[t]{2}{*}{$\overline{0.20}$} & 0.0595 & 0.0284 & 0.057815 & 0.01109 & 0.048 & 0.591 & 1.791 & 2.959 \\
\hline & & 0.0280 & 0.057816 & 0.01113 & 0.048 & 0.582 & & 2.923 \\
\hline \multirow[t]{2}{*}{$\overline{0.30}$} & & 0.0288 & 0.057836 & 0.01155 & 0.037 & 0.975 & 2.118 & $\overline{3.251}$ \\
\hline & & 0.0285 & 0.057836 & 0.01161 & 0.038 & 0.968 & & 3.217 \\
\hline \multicolumn{9}{|c|}{ TABLE VI $(M / R=0.15)$} \\
\hline \multirow[t]{2}{*}{$\overline{0.20}$} & 0.1534 & 0.1303 & 0.140859 & 0.04174 & 0.116 & 0.413 & 1.244 & 2.067 \\
\hline & & 0.1242 & 0.140774 & 0.04194 & 0.117 & 0.395 & & 2.037 \\
\hline \multirow[t]{2}{*}{$\overline{0.30}$} & & 0.1341 & 0.140971 & 0.04268 & 0.092 & 0.682 & 1.477 & $\overline{2.273}$ \\
\hline & & 0.1286 & 0.140874 & 0.04294 & 0.093 & 0.668 & & 2.249 \\
\hline
\end{tabular}

\section{Irrotational case}

For irrotational relativistic configurations, a detailed comparison with Uryu \& Eriguchi results [23,24] is underway 65. For the purpose of the present article, we have compared only the cusp point configuration of a $M / R=0.14$ $\gamma=2$ polytropic sequence as given in the last but one line of Table IV of Ref. [23] and the last line of our Table IV] below. The agreement is very satisfactory: the relative discrepancy is below $0.1 \%$ on $M, 0.4 \%$ on $\Omega M_{\mathrm{B}}, 2.1 \%$ on $J / M^{2}$ and $2.1 \%$ on the semi-diameter $a_{0}$ of the stars [Eq. 129) below]. 


\section{RESULTS FOR $\gamma=2$ POLYTROPES}

The tests of the code being successfully passed, we employ the code to compute an irrotational relativistic sequence based on the polytropic equation of state (105)-(107) with $\gamma=2$. We are using $\kappa=0.0332 \rho_{\text {nuc }}^{-1} c^{2}$, and consider the compactness parameter $M / R=0.14$ at infinite separation. This results in the baryon mass $M_{\mathrm{B}}=1.625 M_{\odot}$.

The computational parameters are as follows: six domains are used, such that (using the notations of Sect. IV A): $N_{\langle 1\rangle}=N_{\langle 2\rangle}=3, M_{\langle 1\rangle}=M_{\langle 2\rangle}=1$, with the same number of coefficients in each domain: $N_{r} \times N_{\theta} \times N_{\varphi}=33 \times 21 \times 20$. The criterion to end the computation of one configuration is set to $\delta H=10^{-7}$. A special treatment has been performed for the closest configuration, because of the existence of a cusp on the stellar surface $\left(\right.$ Sect. IVE): $N_{r} \times N_{\theta} \times N_{\varphi}=$ $25 \times 17 \times 16$ coefficients have been used along with the enthalpy gradient threshold $\chi_{\mathrm{fr}}=0.55$, resulting in a frozen mapping.

In Figs. 19 and 20, the half of the ADM mass and the total angular momentum of the binary system, as defined in Sect. IIID, are shown along the evolutionary sequence. This sequence ends at around $d=37.5 \mathrm{~km}(f=380 \mathrm{~Hz})$ where a cusp appears on the surface of the stars.

One can see from these figures that there is no turning point for the $\gamma=2$ case. This result agrees with that of Uryu \& Eriguchi [23].

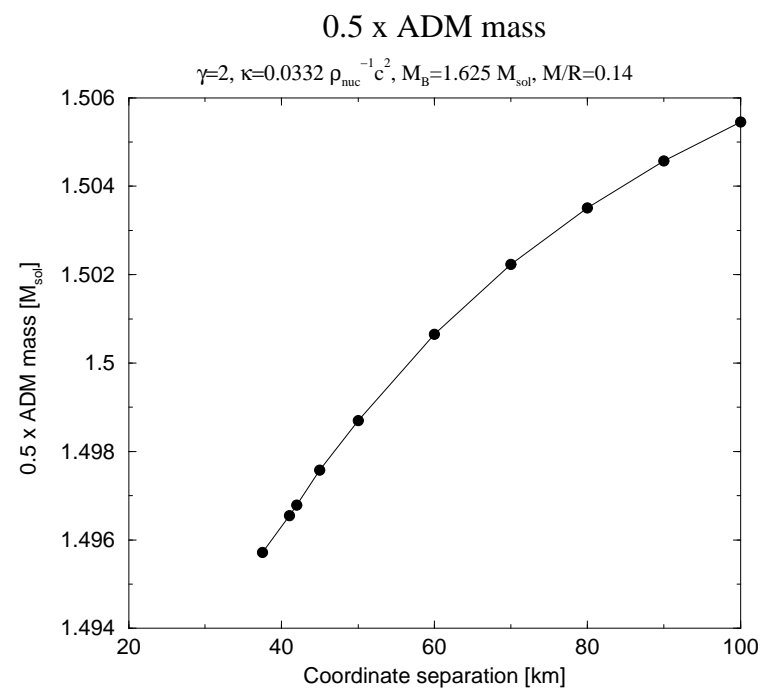

FIG. 19. Half of the ADM mass of the binary system as a function of the coordinate separation for an evolutionary sequence of relativistic irrotational stars.

Total angular momentum

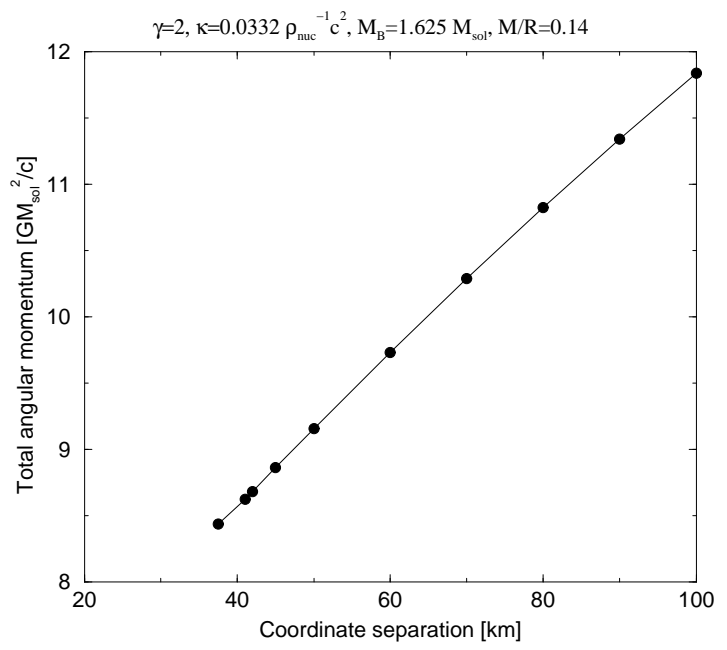

FIG. 20. Same as Fig. 19 but for the total angular momentum. 
An important result of this computation has already been presented in Ref. [21], namely the central energy density remains rather constant (with a slight increase below $0.01 \%$ ) and finally decreases (see Fig. 6). As discussed in the Introduction, this result makes the collapse of the individual neutron stars to black hole very unlikely prior to the merger.

We summarize the results about the sequence in Table III, listing the ADM mass, the total angular momentum, the orbital angular velocity, the axis ratios, and the relative change in central energy density along the quasiequilibrium sequence. Since this is the first table presented for a sequence of relativistic irrotational binary neutron stars, we give a rather large number of digits in order to compare with the results of other works from now on. Note that we are using the following values of the fundamental constants: $G=6.6726 \times 10^{-11} \mathrm{~m}^{3} \mathrm{~kg}^{-1} \mathrm{~s}^{-2}, c=2.99792458 \times 10^{8} \mathrm{~m} \mathrm{~s}^{-1}$ and $M_{\odot}=1.989 \times 10^{30} \mathrm{~kg}$.

TABLE III. Half of ADM mass $M$, total angular momentum $J$, orbital angular velocity $\Omega$, axis ratios, and relative change in central energy density along a $M_{\mathrm{B}}=1.625 M_{\odot}$ quasiequilibrium sequence constructed upon a $\gamma=2$ polytropic EOS. $a_{1}$, $a_{2}$, and $a_{3}$ denote the coordinate lengths parallel to the semi-major axes $x, y$, and $z$, respectively. $a_{1, \text { opp }}$ is the length in the direction opposite to the companion star.

\begin{tabular}{|c|c|c|c|c|c|c|c|c|}
\hline$d[\mathrm{~km}]$ & $0.5 \times M\left[M_{\odot}\right]$ & $J\left[G M_{\odot}^{2} / c\right]$ & $\Omega[\mathrm{rad} / \mathrm{s}]$ & $\Omega /(2 \pi)[\mathrm{Hz}]$ & $a_{2} / a_{1}$ & $a_{3} / a_{1}$ & $a_{1, \mathrm{opp}} / a_{1}$ & $\left(e_{c}-e_{c, \infty}\right) / e_{c, \infty}$ \\
\hline 100 & 1.50545 & 11.8370 & 597.24 & 95.054 & 0.99100 & 0.99367 & 0.99319 & $4.0606 \mathrm{e}-05$ \\
\hline 90 & 1.50457 & 11.3403 & 695.15 & 110.64 & 0.98839 & 0.99139 & 0.99234 & $6.0695 \mathrm{e}-05$ \\
\hline 80 & 1.50351 & 10.8243 & 823.17 & 131.01 & 0.98445 & 0.98788 & 0.99106 & $6.9369 \mathrm{e}-05$ \\
\hline 70 & 1.50223 & 10.2880 & 996.14 & 158.54 & 0.97811 & 0.98210 & 0.98903 & $8.4666 \mathrm{e}-05$ \\
\hline 60 & 1.50065 & 9.73115 & 1239.9 & 197.34 & 0.96687 & 0.97171 & 0.98466 & $3.2735 \mathrm{e}-05$ \\
\hline 50 & 1.49870 & 9.15576 & 1603.5 & 255.20 & 0.94402 & 0.95037 & 0.97369 & $-5.9816 \mathrm{e}-04$ \\
\hline 45 & 1.49758 & 8.86296 & 1858.8 & 295.84 & 0.92226 & 0.92999 & 0.96263 & $-2.0684 \mathrm{e}-03$ \\
\hline 42 & 1.49679 & 8.68172 & 2041.4 & 324.89 & 0.90315 & 0.91100 & 0.95408 & $-4.2246 \mathrm{e}-03$ \\
\hline 41 & 1.49655 & 8.62425 & 2111.0 & 335.98 & 0.89206 & 0.89940 & 0.95076 & $-5.4239 \mathrm{e}-03$ \\
\hline 37.5 & 1.49572 & 8.43623 & 2389.7 & 380.33 & 0.81445 & 0.82752 & 0.91949 & $-1.2238 \mathrm{e}-02$ \\
\hline
\end{tabular}


For comparison purposes with other works, we also give Table IV in which the physical quantities are normalized by using the equation of state constants $\kappa$ and $\gamma$ to set a length scale $R_{\text {poly }}$ according to

$$
R_{\text {poly }}:=\kappa^{\frac{1}{2(\gamma-1)}} .
$$

We can therefore define the same dimensionless quantities as Baumgarte et al. [26] and Uryu \& Eriguchi [23]: $\bar{M}_{\mathrm{B}}=$ $M_{\mathrm{B}} / R_{\text {poly }}, \bar{M}=M / R_{\text {poly }}, \bar{J}=J / R_{\text {poly }}^{2}, \bar{\Omega}=R_{\text {poly }} \Omega, \bar{d}=d / R_{\text {poly }}, \bar{d}_{G}=d_{G} / R_{\text {poly }}, \bar{a}_{0}=a_{0} / R_{\text {poly }}$, where $d_{G}$ is the distance between the "centers of mass" of each stars as defined by Eq. (107) of Uryu \& Eriguchi [23]:

$$
d_{G}:=\left|\frac{1}{M_{\mathrm{B}}^{\langle 1\rangle}} \int_{\text {star } 1} A^{3} \Gamma_{\mathrm{n}} n X d^{3} x-\frac{1}{M_{\mathrm{B}}^{\langle 2\rangle}} \int_{\operatorname{star} 2} A^{3} \Gamma_{\mathrm{n}} n X d^{3} x\right|
$$

and $a_{0}$ is half of the coordinate length of a star along the $X$ axis:

$$
a_{0}=\frac{1}{2}\left|X_{\max }-X_{\min }\right| \text {. }
$$

This latter quantity is denoted $R_{0}$ by Uryu \& Eriguchi [23].

TABLE IV. Dimensionless ADM mass $\bar{M}$, total angular momentum $\bar{J}$, orbital angular velocity $\bar{\Omega}$, and half of the coordinate length along the $X$ axis $\bar{a}_{0}$ along the $\bar{M}_{\mathrm{B}}=0.146202$ quasiequilibrium sequence presented in Table III.

\begin{tabular}{cccccccr}
\hline \hline $\bar{d}$ & $\bar{d}_{G}$ & $0.5 \times \bar{M}$ & $\bar{J}$ & $J / M^{2}$ & $\bar{\Omega}$ & $\Omega M_{\mathrm{B}}$ & $\bar{a}_{0}$ \\
\hline 6.0927 & 6.0924 & 0.135446 & $9.58174 \mathrm{e}-02$ & 1.30573 & $3.2698 \mathrm{e}-02$ & $4.7805 \mathrm{e}-03$ & 0.81089 \\
5.4835 & 5.4830 & 0.135367 & $9.17965 \mathrm{e}-02$ & 1.25239 & $3.8058 \mathrm{e}-02$ & $5.5642 \mathrm{e}-03$ & 0.80934 \\
4.8742 & 4.8736 & 0.135272 & $8.76195 \mathrm{e}-02$ & 1.19708 & $4.5067 \mathrm{e}-02$ & $6.5889 \mathrm{e}-03$ & 0.80770 \\
4.2649 & 4.2642 & 0.135156 & $8.32782 \mathrm{e}-02$ & 1.13973 & $5.4536 \mathrm{e}-02$ & $7.9733 \mathrm{e}-03$ & 0.80623 \\
3.6556 & 3.6546 & 0.135014 & $7.87708 \mathrm{e}-02$ & 1.08031 & $6.7883 \mathrm{e}-02$ & $9.9246 \mathrm{e}-03$ & 0.80542 \\
3.0464 & 3.0447 & 0.134839 & $7.41131 \mathrm{e}-02$ & 1.01907 & $8.7788 \mathrm{e}-02$ & $1.2835 \mathrm{e}-02$ & 0.80796 \\
2.7417 & 2.7396 & 0.134738 & $7.17429 \mathrm{e}-02$ & 0.98796 & $1.0177 \mathrm{e}-01$ & $1.4879 \mathrm{e}-02$ & 0.81489 \\
2.5589 & 2.5565 & 0.134667 & $7.02758 \mathrm{e}-02$ & 0.96878 & $1.1176 \mathrm{e}-01$ & $1.6339 \mathrm{e}-02$ & 0.82583 \\
2.4980 & 2.4954 & 0.134645 & $6.98107 \mathrm{e}-02$ & 0.96268 & $1.1557 \mathrm{e}-01$ & $1.6897 \mathrm{e}-02$ & 0.83271 \\
2.2848 & 2.2810 & 0.134571 & $6.82887 \mathrm{e}-02$ & 0.94273 & $1.3083 \mathrm{e}-01$ & $1.9127 \mathrm{e}-02$ & 0.88016 \\
\hline \hline
\end{tabular}


Finally, let us show some figures about metric, density, and internal velocity fields. The lapse function $N$ is represented in Fig. 21. The coordinate system is the $(X, Y, Z)$ one defined in Sect. IV A and the coordinate separation is $41 \mathrm{~km}$ (last but one line in Tables III and IV). At this separation, the central value of $N$ of each star is 0.6416 .

The shift vector $\mathbf{N}$ of non-rotating coordinates [defined by Eq. (47)] is shown in Fig. 22. The plot is a cross section of the orbital plane.

The $K^{X X}, K^{X Y}$, and $K^{Y Y}$ components of the extrinsic curvature tensor of the hypersurfaces $t=$ const are shown in Fig. 23. The values in the figures are multiplied by the square conformal factor $A^{2}$, and the plots are cross section of the orbital plane.
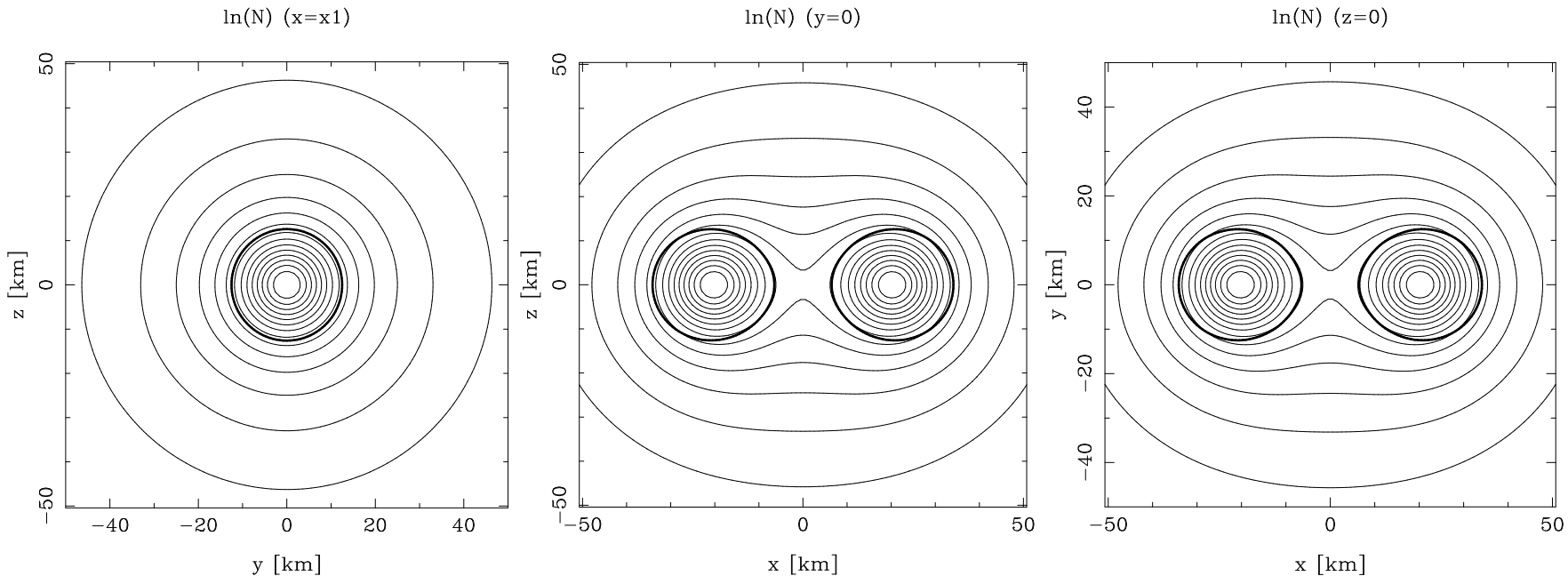

FIG. 21. Isocontour of the gravitational potential $\nu$ (logarithm of the lapse function $N$ ) when the coordinate separation is $41 \mathrm{~km}$. The plots are cross sections of the $X=-20.5 \mathrm{~km}, Y=0$, and $Z=0$ planes. The thick solid lines denote the surfaces of the stars.

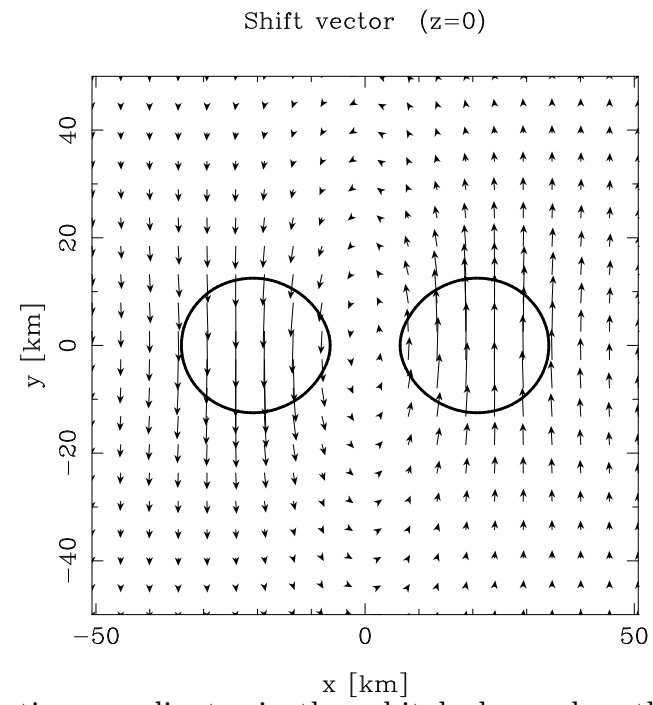

FIG. 22. Shift vector $\mathbf{N}$ of non-rotating coordinates in the orbital plane when the coordinate separation is $41 \mathrm{~km}$. The thick solid lines denote the surfaces of the stars. 
$\mathrm{A}^{2} \mathrm{~K}^{\mathrm{xx}}(\mathrm{z}=0)$

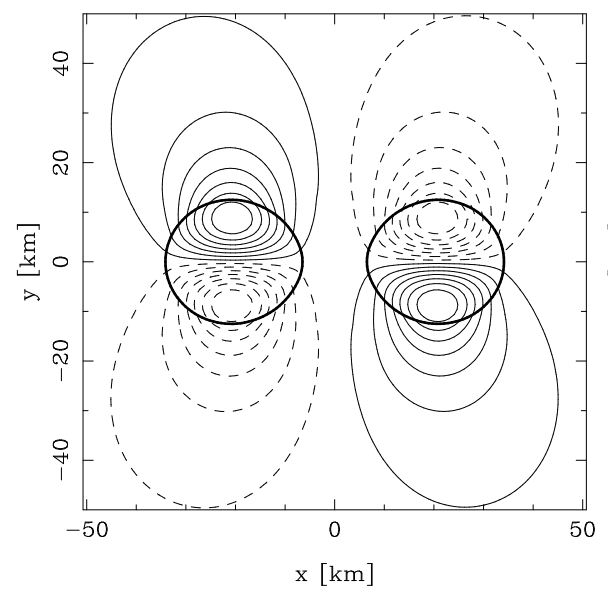

$\mathrm{A}^{2} \mathrm{~K}^{\mathrm{xy}}(\mathrm{z}=0)$

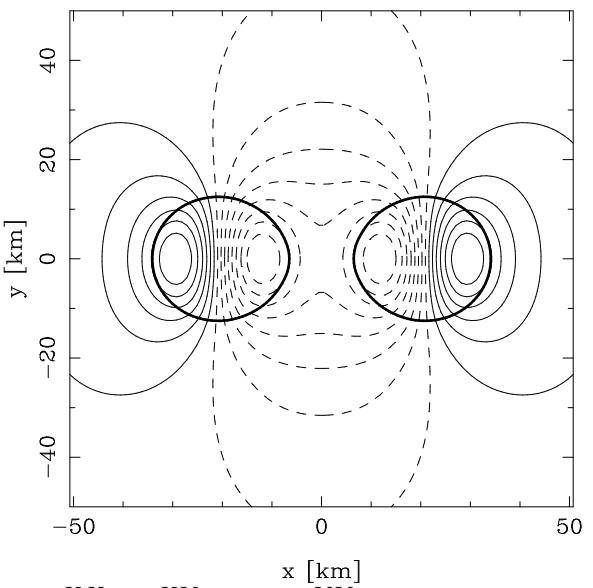

$\mathrm{A}^{2} \mathrm{~K}^{\mathrm{yy}}(\mathrm{z}=0)$

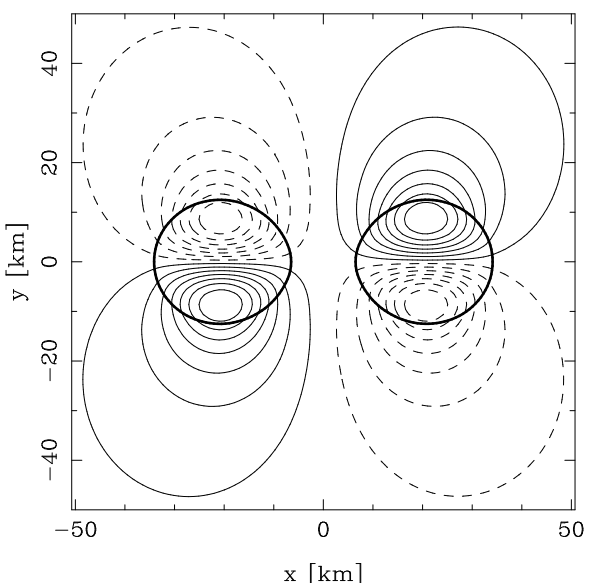

FIG. 23. Isocontours of the components $K^{X X}, K^{X Y}$, and $K^{Y Y}$ (multiplied by $A^{2}$ ) of the extrinsic curvature tensor when the coordinate separation is $41 \mathrm{~km}$. The plots are cross sections of the orbital plane $(Z=0)$. Solid (resp. dashed) lines denotes positive (resp. negative) values. The thick solid lines mark the surfaces of the stars.

The baryon density field in the fluid frame is shown in Fig. 24. The plots are cross sections of $Z=0$ and $Y=0$ planes.

Finally, we show in Fig. 25 the internal velocity field in the co-orbiting frame, or more precisely the orthogonal projection in the $\Sigma_{t}$ hypersurface of the vector field $\mathbf{V}$ given by Eq. (30). Note that this vector field is tangent to the surface of the stars, as it should be.
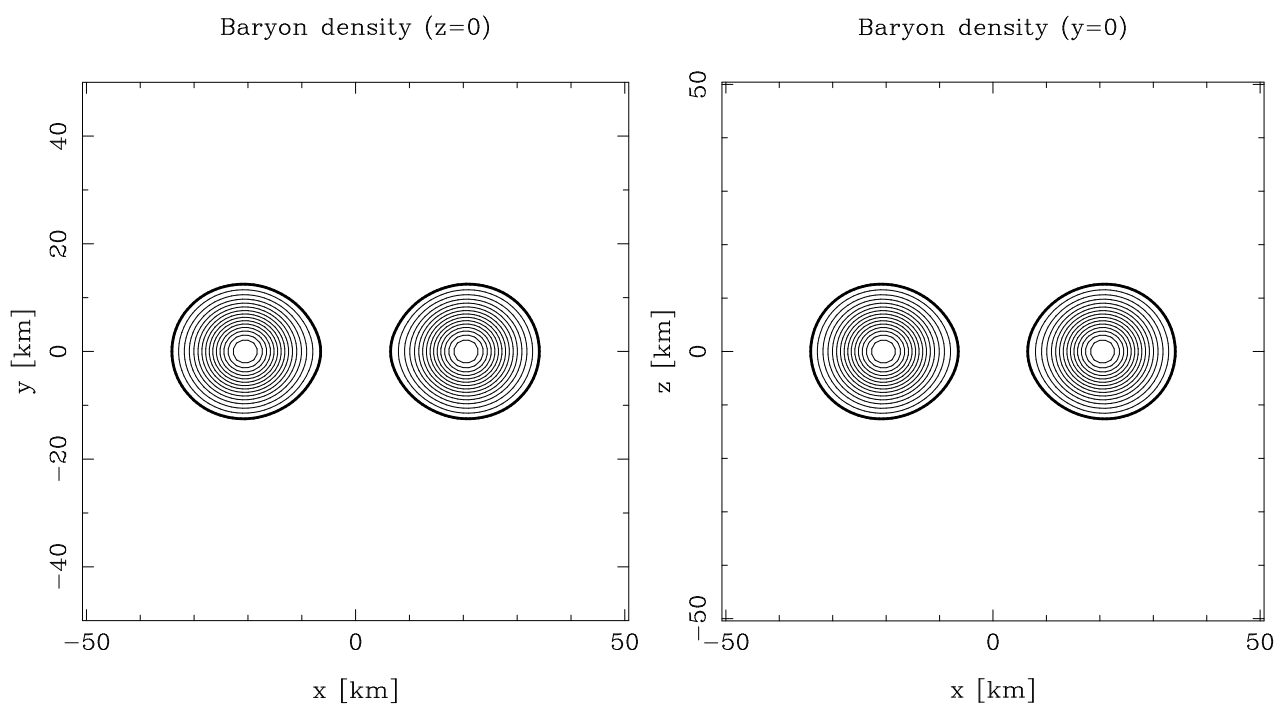

FIG. 24. Isocontours of the fluid proper baryon density $n$ when the coordinate separation is $41 \mathrm{~km}$. The plots are cross sections of $Z=0$ and $Y=0$ planes. The thick solid lines denote the surfaces of the stars. 


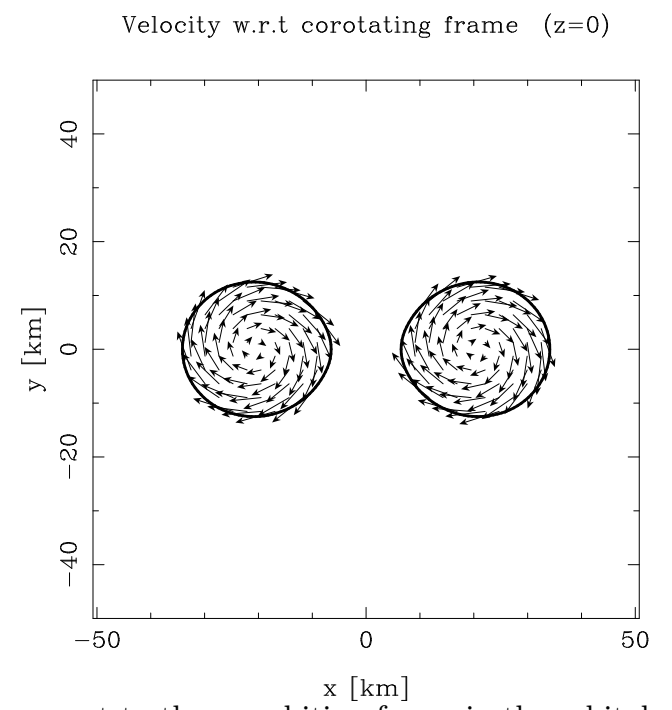

FIG. 25. Internal velocity field with respect to the co-orbiting frame in the orbital plane when the coordinate separation is $41 \mathrm{~km}$. The thick solid lines denote the surfaces of the stars.

\section{DISCUSSION}

\section{A. Comparison with other numerical methods}

The numerical method presented in this article is the only method for computing relativistic binaries in which the computational domains extends to infinity, thereby enabling to impose exact boundary conditions on the gravitational field equations. All the other methods [26,22,23] employ finite computational boxes. Our experience from calculations of single rotating neutron stars show that the finite size of the computational domain can result in some loss of accuracy (see Ref. [66] for a discussion of this point).

Besides, thanks to the splitting of the metric quantities in a part described on the domains centered on star 1 and a part described on the domains centered on star 2, we can describe without any loss of accuracy very distant stars. In fact we can recover the spherical limit when the stars have very large separations, contrary to all other numerical methods which are losing resolution when the stars are put farther apart (see for instance the discussion in Sect. V.A of Ref. 26]).

We are using surface-fitted spherical coordinates, which by construction are well adapted to describe the stellar fluid interiors. As it can be seen on Figs. 21 23, these coordinate systems, which are centered on one of the two stars, are also well adapted to the description of the metric quantities, because these latter are maximum at the location of the stars. Baumgarte et al. 26] and Marronetti et al. 222 use instead Cartesian coordinates in a single domain ("box"). Closer to our approach, Uryu \& Eriguchi [23] developed a multi-domain method with surface-fitted spherical coordinates, which enable them to treat precisely the fluid interiors of the stars. However, for the gravitational field they use a single spherical coordinate system which is centered at the system center of mass.

As far as irrotational binaries are concerned, we payed a special attention to the resolution of the equation for the velocity potential $\Psi$. First we solve numerically only for a small part $\Psi_{0}$ of $\Psi$, thereby reducing the numerical error. Second we let appear in the equation for $\Psi_{0}$ a partial differential operator which is invertible and give as a unique solution that with the correct behavior at the stellar surface (velocity field tangent to the surface in the co-orbiting frame). The equation for $\Psi$ is instead solved as a Poisson equation $\Delta \Psi=$ source with a boundary condition at the stellar surface by Uryu \& Eriguchi [23] and Marronetti et al. 22, 31]. Note that Marronetti et al. performs only an approximate treatment of the boundary condition, which amounts to considering that the surface of the star is an exact sphere. This is of course not valid for close configurations. On the contrary, thanks to the introduction of surface-fitted coordinates, Uryu \& Eriguchi [23] have been able to treat the boundary condition exactly.

\section{B. Tests passed by the code}

We have performed extensive tests of the numerical code. In particular, we have shown that, in the Newtonian limit, our numerical results coincides with the semi-analytical solutions recently obtained by Taniguchi \& Nakamura 
63, 64 for compressible polytropic stars. The only discrepancies appeared to be due to missing higher order terms in Taniguchi \& Nakamura's solutions and not to some inaccuracy of the numerical code.

Regarding relativistic configurations, no analytical solutions was available to compare with. In this case, we checked only by comparing with previous numerical solutions, namely that of Baumgarte et al. [26] for synchronized binaries and Uryu \& Eriguchi 23] for irrotational binaries. The agreement is of the order of 1\%. For the astrophysically relevant case of irrotational relativistic binaries, a detailed comparison with Uryu \& Eriguchi code [23] is underway 65.

\section{Future prospects}

We are currently using the method described in this article to compute models of close binary neutron stars with various equations of states: polytropic EOS with various polytropic indices, dense matter EOS resulting from recent nuclear physics calculations. In particular, we are studying how parameters like the frequency location of the innermost stable orbit (if any) depends on the equation of state, in order to help in the interpretation of gravitational wave signals from coalescing neutron star binaries. The results of these studies will be published elsewhere [67.

\section{ACKNOWLEDGMENTS}

We would like to thank Luc Blanchet, Brandon Carter, Pedro Marronetti, John Stewart and Koji Uryu for useful discussions and Masaru Shibata for suggesting us to plot Fig. 12. The code development and the numerical computations have been performed on SGI workstations purchased thanks to a special grant from the C.N.R.S.

\section{APPENDIX A: LINK WITH TEUKOLSKY'S AND SHIBATA'S FORMULATIONS}

The first integral of motion for quasiequilibrium irrotational binaries derived by Teukolsky [19] is (cf. his Eq. (57), re-written within our notations (8)

$$
N\left(h^{2}+\mathbf{D} \Psi \cdot \mathbf{D} \Psi\right)^{1 / 2}+\mathbf{B} \cdot \mathbf{D} \Psi=C,
$$

where $C$ is some constant. At first glance, this might look quite different from our first integral of motion (34). However, if we substitute Eq. (29) for $\Gamma$ in the exponential form of our first integral (i.e. Eq. (33)) we get

$$
h N \Gamma_{\mathrm{n}}\left(1-\mathbf{U} \cdot \mathbf{U}_{0}\right)=\text { const. }
$$

By means of Eqs. (31) and (24), this equations becomes

$$
h N \Gamma_{\mathrm{n}}+\mathbf{B} \cdot \mathbf{D} \Psi=\text { const. }
$$

Finally, if we substitute Eq. (32) for $\Gamma_{\mathrm{n}}$ is this relation, we recover Eq. (A1). In particular this shows that the constant in the right-hand side of Eq. (33) is nothing but the constant denoted $C$ by Teukolsky [19].

The first integral of motion for quasiequilibrium irrotational binaries derived by Shibata [20] is (cf. his Eq. (2.18), re-written within our notations 9])

$$
\frac{h}{\lambda}+\mathbf{S} \cdot \mathbf{D} \Psi=\text { const }
$$

where $\lambda$ and $\mathbf{S}$ are defined by the following decomposition of the fluid 4-velocity in a part along the Killing vector $\mathbf{l}$ and a part parallel to the hypersurface $\Sigma_{t}$ :

$$
\mathbf{u}=\lambda(\mathbf{l}+\mathbf{S}) \quad \text { with } \quad \mathbf{n} \cdot \mathbf{S} .
$$

\footnotetext{
${ }^{8}$ Our shift vector B is the negative of Teukolsky's B.

${ }^{9}$ Our $\lambda$ and $\mathbf{S}$ are denoted respectively by $u^{0}$ and $V^{i}$ by Shibata [20].
} 
Now, substituting Eq. (3) for $\mathbf{l}$ in this relation and using Eq. (27), we get

$$
\mathbf{S}=\frac{1}{\lambda} \Gamma_{\mathrm{n}} \mathbf{U}+\mathbf{B}=\frac{1}{\lambda h} \mathbf{D} \Psi+\mathbf{B},
$$

where the second equality follows from Eq. (31). Inserting Eq. (A6) into (A5) and using the normalization relation $\mathbf{u} \cdot \mathbf{u}=-1$ results in the following expression for $\lambda$ :

$$
\lambda=\frac{1}{h N}\left(h^{2}+\mathbf{D} \Psi \cdot \mathbf{D} \Psi\right)^{1 / 2} .
$$

Finally substituting Eq. (A7) for $\lambda$ and Eq. (A6) for $\mathbf{S}$ into Shibata's first integral of motion (A4) result in Teukolsky's form of the integral of motion (Eq. (A1) above), which shows the equivalence of the various formulations.

\section{APPENDIX B: NUMERICAL METHOD TO SOLVE THE ELLIPTIC EQUATION FOR THE VELOCITY POTENTIAL}

Equation (63) for the part $\Psi_{0}$ of the velocity potential can be written

$$
a \underline{\Delta} \Psi_{0}+b^{i} \bar{\nabla}_{i} \Psi_{0}=\sigma,
$$

with

$$
\begin{gathered}
a:=\zeta H, \\
b^{i}:=(1-\zeta H) \bar{\nabla}^{i} H+\zeta H \bar{\nabla}^{i} \beta,
\end{gathered}
$$

and

$$
\sigma:=\left(W^{i}-W_{0}^{i}\right) \bar{\nabla}_{i} H+\zeta H\left(W_{0}^{i} \bar{\nabla}_{i}(H-\beta)+\frac{W^{i}}{\Gamma_{\mathrm{n}}} \bar{\nabla}_{i} \Gamma_{\mathrm{n}}\right) .
$$

Equation (B1) is not merely a Poisson type equation for $\Psi_{0}$ because the coefficient $a$ vanishes at the surface of the star. It therefore deserves a special treatment. In the works of Marronetti et al. [22,31] and Uryu \& Eriguchi [23], Eq. (B1) is recast as a Poisson equation ${ }^{10} \Delta \Psi_{0}=$ source, dividing both sides of Eq. (B1) by $a$. In order that the right-hand side be regular, one must then impose as a boundary condition $b^{i} \bar{\nabla}_{i} \Psi_{0}-\sigma=0$ at the surface of the star. We choose here a different approach, considering that the operator in Eq. (B1) is not the Laplacian but instead the operator

$$
L \Psi_{0}:=\alpha\left(1-\xi^{2}\right) \Delta_{\xi} \Psi_{0}+\beta \xi \frac{\partial \Psi_{0}}{\partial \xi},
$$

where $\alpha$ and $\beta$ are two constants, $\xi \in[0,1]$ is the computational radial coordinate introduced in the mapping (74), and $\Delta_{\xi}$ is an operator which, expressed in terms of the computational coordinates $\left(\xi, \theta^{\prime}, \varphi^{\prime}\right)$ has the same structure than the Laplacian operator:

$$
\Delta_{\xi} \Psi_{0}:=\frac{1}{\xi^{2}} \frac{\partial}{\partial \xi}\left(\xi^{2} \frac{\partial \Psi_{0}}{\partial \xi}\right)+\frac{1}{\xi^{2} \sin \theta^{\prime}} \frac{\partial}{\partial \theta^{\prime}}\left(\sin \theta^{\prime} \frac{\partial \Psi_{0}}{\partial \theta^{\prime}}\right)+\frac{1}{\xi^{2} \sin ^{2} \theta^{\prime}} \frac{\partial^{2} \Psi_{0}}{\partial \varphi^{\prime 2}}
$$

Here we assume that there is only one domain covering the star, i.e. that $M_{\langle 1\rangle}=M_{\langle 2\rangle}=1$, so that the surface of the star is given by $\xi=1$. Equation (B1) is then re-written as

$$
L \Psi_{0}=\sigma+\alpha\left(1-\xi^{2}\right) \Delta_{\xi} \Psi_{0}-a \underline{\Delta} \Psi_{0}+\beta \xi \frac{\partial \Psi_{0}}{\partial \xi}-b^{i} \bar{\nabla}_{i} \Psi_{0} .
$$

\footnotetext{
${ }^{10}$ These authors are using $\Psi$ and not $\Psi_{0}$ as the unknown function, but this has no consequence on the following discussion.
} 
The basic idea is to solve this equation by iterations, considering the whole right-hand as a source term and using the previous step value of $\Psi_{0}$ in it. One must also choose the constants $\alpha$ and $\beta$ so that the term $\alpha\left(1-\xi^{2}\right) \Delta_{\xi} \Psi_{0}$ [resp. $\left.\beta \xi \partial \Psi_{0} / \partial \xi\right]$ is as close as possible to $a \underline{\Delta} \Psi_{0}$ [resp. $\left.b^{i} \bar{\nabla}_{i} \Psi_{0}\right]$. We opt for the following choices:

$$
\alpha=\left.a\left(\frac{\partial r}{\partial \xi}\right)^{2}\right|_{\xi=0}
$$

and

$$
\beta=-\max \left|b^{r}\left(\frac{\partial r}{\partial \xi}\right)^{2}\right|,
$$

where $b^{r}$ is the $r$-component of the vector $b^{i}$. In solving Eq. (B7) by iterations, we introduce the following relaxation

$$
\Psi_{0}^{J} \leftarrow \lambda \Psi_{0}^{J}+(1-\lambda) \Psi_{0}^{J-1}
$$

where $J$ (resp. $J-1$ ) labels the current step (resp. previous step) and $\lambda$ is the relaxation factor, typically chosen to be 0.5 .

At each step of the iteration, Eq. (B7) is solved by the following spectral method. First an expansion in spherical harmonics $Y_{\ell}^{m}\left(\theta^{\prime}, \varphi^{\prime}\right)$ is performed, so that Eq. (B7) becomes equivalent to a set of ordinary differential equations [one equation for each couple $(\ell, m)]$ :

$$
L_{\ell} f(\xi)=s(\xi)
$$

where $f(\xi)$ and $s(\xi)$ are the $(\ell, m)$ coefficient of $\Psi_{0}$ and of the whole right-hand side of (B7) respectively and $L_{\ell}$ is the following differential operator:

$$
L_{\ell} f:=\alpha\left(1-\xi^{2}\right)\left[\frac{d^{2} f}{d \xi^{2}}+\frac{2}{\xi} \frac{d f}{d \xi}-\frac{\ell(\ell+1)}{\xi^{2}} f\right]+\beta \xi \frac{d f}{d \xi} .
$$

Since the source $s(\xi)$ vanishes for $\ell=0$, we treat only the case $\ell>0$. Regularity properties at the origin $(\xi=0)$ imply that $f(\xi)$ and $s(\xi)$ should be expandable in even (resp. odd) Chebyshev polynomials $T_{n}(\xi)$ for $\ell$ even (resp. odd). Due to the division by $\xi$ and $\xi^{2}$, the differential operator $L_{\ell}$ is singular on Chebyshev polynomials at $\xi=0$, except for $\ell=1$. Therefore, instead of Chebyshev polynomials, we use the following polynomials $P_{n}(\xi)$ as a expansion basis for $f\left(N\right.$ is the total number of coefficients in the Chebyshev expansions, denoted $N_{r}^{\langle a\rangle}(0)$ in Sect. [V B)

- for $\ell$ even : $P_{n}(\xi):=T_{2 n}(\xi)+T_{2 n+2}(\xi)=2 \xi T_{2 n+1}(\xi), \quad 0 \leq n \leq N-2$;

- for $\ell=1: P_{n}(\xi):=T_{2 n+1}(\xi), \quad 0 \leq n \leq N-1$;

- for $\ell$ odd and $\ell>1: P_{n}(\xi):=(2 n+3) T_{2 n+1}(\xi)+(2 n+1) T_{2 n+3}(\xi), \quad 0 \leq n \leq N-2$.

The operator on the left-hand side of Eq. (B11) is regular for each of the polynomials $P_{n}(\xi)$ (such a basis is called a Galerkin basis).

We thus consider the differential operator $L_{\ell}$ acting

- for $\ell$ even : from the $N-1$ dimensional vectorial space span by the polynomials $P_{n}(\xi)(0 \leq n \leq N-2)$ to the $N-1$ dimensional vectorial space span by the polynomials $T_{2 n}(\xi)(0 \leq n \leq N-2)$;

- for $\ell=1$ : from the $N$ dimensional vectorial space span by the polynomials $P_{n}(\xi)=T_{2 n+1}(\xi)(0 \leq n \leq N-1)$ to itself;

- for $\ell$ odd and $\ell>1$ : from the $N-1$ dimensional vectorial space span by the polynomials $P_{n}(\xi)(0 \leq n \leq N-2)$ to the $N-1$ dimensional vectorial space span by the polynomials $T_{2 n+1}(\xi)(0 \leq n \leq N-2)$.

The operator $L_{\ell}$ is then one-to-one (isomorphism) between these vectorial spaces. This means that the only homogeneous solution is zero. Otherwise stated, for each $\ell$ there is a unique solution to Eq. (B11) in the vectorial space spans by the $P_{n}(\xi)$ 's. To find this solution, we transform the matrix $A_{i j}$ of $L_{\ell}$ in the above bases into a banded matrix by means of the following linear combinations: 
- for $\ell$ even :

$$
\begin{aligned}
& \bar{A}_{i j}=\frac{1}{i+1}\left[A_{i j}-A_{(i+1) j}\right] \quad \text { for } 0 \leq i \leq N-3 ; \\
& \tilde{A}_{i j}=\bar{A}_{i j}-\bar{A}_{(i+2) j} \quad \text { for } 0 \leq i \leq N-5 .
\end{aligned}
$$

- for $\ell$ odd :

$$
\begin{aligned}
& \bar{A}_{i j}=\frac{1}{i+1}\left[\left(1+\delta_{0}^{i}\right) A_{i j}-A_{(i+2) j}\right] \quad \text { for } 0 \leq i \leq N-3 \\
& \tilde{A}_{i j}=\bar{A}_{i j}-\bar{A}_{(i+2) j} \quad \text { for } 0 \leq i \leq N-5 .
\end{aligned}
$$

Since the resulting matrix $\tilde{A}_{i j}$ has at most 5 bands, the linear system is easily and CPU-efficiently solved to get the coefficients of the solution $f$ in the basis of the polynomials $P_{n}(\xi)$. A simple combination is then performed on these coefficients to get the coefficients on the usual Chebyshev bases.

A very discriminating test of this numerical technique, namely the evaluation of the tiny $z$-component of the velocity field resulting from $\Psi$, is presented in Figs. 13 .15 (Sect. V B 2).

[1] F.A. Rasio and S.L. Shapiro, Class. Quantum Grav. 16, R1 (1999).

[2] L. Blanchet and B.R. Iyer, in Gravitation and relativity: at the turn of the millennium, edited by N. Dadhich and J. Narlikar, (Inter-University Centre for Astronomy and Astrophysics, Pune, 1998), p. 403.

[3] A. Buonanno and T. Damour, Phys. Rev. D 59, 084006 (1999).

[4] K. Taniguchi, Prog. Theor. Phys. 101, 283 (1999).

[5] K. Oohara and T. Nakamura, in Relativistic Gravitation and Gravitational Radiation, edited by J.A. Marck and J.P. Lasota (Cambridge University Press, Cambridge, England, 1997), p. 309.

[6] J.R. Wilson and G.J. Mathews, Phys. Rev. Lett. 75, 4161 (1995).

[7] J.R. Wilson, G.J. Mathews, and P. Marronetti, Phys. Rev. D 54, 1317 (1996).

[8] M. Shibata, Prog. Theor. Phys. 101, 251 (1999).

[9] M. Shibata, Prog. Theor. Phys. 101, 1199 (1999).

[10] M. Shibata, Phys. Rev. D 60, 104052 (1999).

[11] M. Shibata and K. Uryu, Phys. Rev. D 61, 064001 (2000).

[12] W.-M. Suen, Prog. Theor. Phys. Suppl. 136, 251 (1999).

[13] J.A. Font, M. Miller, W.-M. Suen, and M. Tobias, Phys. Rev. D 61, 044011 (2000).

[14] K. Oohara and T. Nakamura, Prog. Theor. Phys. Suppl. 136, 270 (1999).

[15] J.W. York, in Sources of gravitational radiation, edited by L. Smarr (Cambridge University Press, Cambridge, England, 1979), p. 83.

[16] E. Seidel and W.-M. Suen, J. Comput. Appl. Math. 109, 493 (1999).

[17] S. Bonazzola, E. Gourgoulhon, and J-A. Marck, Phys. Rev. D 56, 7740 (1997).

[18] H. Asada, Phys. Rev. D 57, 7292 (1998).

[19] S.A. Teukolsky, Astrophys. J. 504, 442 (1998).

[20] M. Shibata, Phys. Rev. D 58, 024012 (1998).

[21] S. Bonazzola, E. Gourgoulhon, and J-A. Marck, Phys. Rev. Lett. 82, 892 (1999).

[22] P. Marronetti, G.J. Mathews, and J.R. Wilson, Phys. Rev. D 60, 087301 (1999).

[23] K. Uryu and Y. Eriguchi, Phys. Rev. D 61, 124023 (2000).

[24] K. Uryu, M. Shibata, and Y. Eriguchi, Phys. Rev. D 62, 104015 (2000).

[25] T.W. Baumgarte, G.B. Cook, M.A. Scheel, S.L. Shapiro, and S.A. Teukolsky, Phys. Rev. Lett. 79, 1182 (1997).

[26] T.W. Baumgarte, G.B. Cook, M.A. Scheel, S.L. Shapiro, and S.A. Teukolsky, Phys. Rev. D 57, 7299 (1998).

[27] P. Marronetti, G.J. Mathews, and J.R. Wilson, Phys. Rev. D 58, 107503 (1998).

[28] C.S. Kochanek, Astrophys. J. 398, 234 (1992).

[29] L. Bildsten and C. Cutler, Astrophys. J. 400, 175 (1992).

[30] S. Bonazzola, E. Gourgoulhon, and J-A. Marck, Phys. Rev. D 58, 104020 (1998).

[31] P. Marronetti, G.J. Mathews, and J.R. Wilson, in Proceedings of 19th Texas Symposium on Relativistic Astrophysics and Cosmology (Paris, France, 14-18 décembre 1998) (CD-ROM version), edited by E. Aubourg, T. Montmerle, J. Paul \& P. Peter (CEA/DAPNIA, Gif sur Yvette, France, 2000). 
[32] G.J. Mathews, P. Marronetti, and J.R. Wilson, Phys. Rev. D 58, 043003 (1998).

[33] E.E. Flanagan, Phys. Rev. Lett. 82, 1354 (1999).

[34] G.J. Mathews and J.R. Wilson, Phys. Rev. D 61, 127304 (2000).

[35] L. Blanchet, T. Damour, B.R. Iyer, C.M. Will, and A.G. Wiseman, Phys. Rev. Lett. 74, 3515 (1995).

[36] P.C. Peters, Phys. Rev. 136, B1224 (1964).

[37] G.W. Gibbons and J.M. Stewart, in Classical General Relativity, edited by W.B. Bonnor, J.N. Islam and M.A.H. MacCallum (Cambridge University Press, Cambridge, England, 1983), p. 77.

[38] J.R. Wilson and G.J. Mathews, in Frontiers in numerical relativity, edited by C.R. Evans, L.S. Finn and D.W. Hobill (Cambridge University Press, Cambridge, England, 1989), p. 306.

[39] A. Lichnerowicz, Relativistic hydrodynamics and magnetohydrodynamics (Benjamin, New York, 1967).

[40] B. Carter, in Active Galactic Nuclei, edited by C. Hazard and S. Mitton (Cambridge University Press, Cambridge, England, 1979), p. 273.

[41] R.H. Boyer, Proc. Cambridge Phil. Soc. 61, 527 (1965).

[42] L.D. Landau and E.M. Lifchitz, Mécanique des fluides (Mir, Moscow, 1989).

[43] K. Uryu and Y. Eriguchi, Astrophys. J. Suppl. 118, 563 (1998).

[44] G.B. Cook, Phys. Rev. D 50, 5025 (1994).

[45] S. Brandt and B. Brügmann, Phys. Rev. Lett. 78, 3606 (1997).

[46] T.W. Baumgarte, Phys. Rev. D 62, 024018 (2000).

[47] H.P. Pfeiffer, S.A. Teukolsky, and G.B. Cook, Phys. Rev. D 62, 104018 (2000).

[48] G.B. Cook, S.L. Shapiro, and S.A. Teukolsky, Phys. Rev. D 53, 5533 (1996).

[49] L. Blanchet, G. Faye, and B. Ponsot, Phys. Rev. D 58, 124002 (1998).

[50] S. Bonazzola, E. Gourgoulhon, and J-A. Marck, in Proceedings of 19th Texas Symposium on Relativistic Astrophysics and Cosmology (Paris, France, 14-18 décembre 1998) (CD-ROM version), edited by E. Aubourg, T. Montmerle, J. Paul \& P. Peter (CEA/DAPNIA, Gif sur Yvette, France, 2000).

[51] C.W. Misner, K.S. Thorne, and J.A. Wheeler, Gravitation (Freeman, New York, 1973).

[52] J.M. Bowen and J.W. York, Phys. Rev. D 21, 2047 (1980).

[53] J.W. York, in Essays in General Relativity, a Festschrift for Abraham Taub, edited by F.J. Tipler (Academic Press, New York, 1980), p. 39.

[54] C. Canuto, M.Y. Hussaini, A. Quarteroni, T.A. Zang, Spectral methods in fluid dynamics, (Springer-Verlag, Berlin, 1988).

[55] S. Bonazzola, E. Gourgoulhon, and J-A. Marck, J. Comp. Appl. Math. 109, 433 (1999).

[56] P. Grandclément, S. Bonazzola, E. Gourgoulhon, and J.-A. Marck, J. Comp. Phys., submitted (preprint: gr-qc/0003072).

[57] L.E. Kidder, Phys. Rev. D 52, 821 (1995).

[58] K. Oohara, T. Nakamura, and M. Shibata, Prog. Theor. Phys. Suppl. 128, 183 (1997).

[59] J-A. Marck, E. Gourgoulhon, P. Grandclément, and J. Novak, Langage Objet pour la RElativité NumériquE - library documentation, in preparation.

[60] S. Chandrasekhar : Elliposoidal figures of equilibrium (Yale University Press, New Haven, 1969).

[61] M.L. Aizenman, Astrophys. J. 153, 511 (1968).

[62] D. Lai, F.A. Rasio, and S.L. Shapiro, Astrophys. J. Suppl. 88, 205 (1993).

[63] K. Taniguchi and T. Nakamura, Phys. Rev. Lett. 84, 581 (2000).

[64] K. Taniguchi and T. Nakamura, Phys. Rev. D 62, 044040 (2000).

[65] E. Gourgoulhon and K. Uryu, in preparation.

[66] T. Nozawa, N. Stergioulas, E. Gourgoulhon, and Y. Eriguchi, Astron. Astrophys. Suppl. Ser. 132, 431 (1998).

[67] K. Taniguchi, E. Gourgoulhon, P. Granclément and S. Bonazzola, in preparation. 